

\title{
Un lemme de Morse pour les surfaces convexes
}

\author{
François LABOURIE *
}

24 octobre 2018

\section{Introduction}

Le lemme de Morse pour les géodésiques affirme que dans une variété à courbure strictement négative et géométrie bornée, toute quasi-géodésique est à une distance bornée d'une géodésique.

D’un point de vue dynamique, il est une version géométrique du "Shadowing Lemma". Il a pour conséquence les trois propriétés hyperboliques du flot géodésique d'une variété compacte à courbure strictement négative, vu ici un feuilletage de dimension 1 :

(i) l'ensemble des feuilles compactes est dense,

(ii) une feuille générique est dense,

(iii) les flots géodésiques de deux variétés à courbure négative proches sont conjugués.

Le but de notre d'article est d'énoncer un lemme de Morse pour les $k$-surfaces dans les variétés de dimension 3 à courbure négative et d'en tirer des conséquences analogues.

Définissons brièvement une $k$-surface, où $k \in] 0,1[$, dans une variété $M$ de dimension 3 à courbure plus petite que -1 : il s'agit d'une surface dont la courbure extrinsèque, c'est-à-dire le produit des courbures principales, vaut $k$. Nos hypothèses sur $k$ entraînent qu'une $k$-surface est localement convexe et à courbure intrinsèque strictement négative. Nous nous intéresserons principalement aux $k$-surfaces non

*l'auteur remercie l'Institut Universitaire de France 
compactes ( elles le sont toutes si $M$ est simplement connexe ) et complètes dans un certain sens ( voir 2.2 pour des précisions). Du point de vue analytique, il s'agit d'un problème elliptique, que nous décrivons comme de Monge-Ampère au sens de 3.

Le lemme de Morse pour les surfaces convexes nous dit en particulier que toute surface localement convexe à courbure extrinsèque plus grande que $k$, ayant de bonnes propriétés à l'infini, est à distance bornée d'une $k$-surface. Il résoud en particulier une sorte de problème de Plateau pour les $k$-surfaces. Malheureusement, pour énoncer correctement ce lemme, il nous faut introduire des définitions techniques qui sortent du cadre de cette présentation (voir 2).

Nous allons présenter maintenant deux types d'application de ce lemme de Morse. Nous étudierons les problèmes de Plateau asymptotiques et les propriétés hyperboliques de l'espace des $k$-surfaces. Ces propriétés hyperboliques, qui font apparaître l'espace des $k$-surfaces comme une généralisation du flot géodésique, sont la motivation essentielle de notre travail.

Pour énoncer de façon simple nos résultats, nous supposerons dans cette introduction que $M$ est le revêtement universel d'une variété $N$ compacte.

\subsection{Problème de Plateau asymptotique}

Soit $i$ un homéomorphisme local d'une surface $S$ dans le bord à l'infini, $\partial_{\infty} M$, de $M$. Une solution du problème de Plateau asymptotique défini par $(i, S)$, est une immersion $f$ de $S$ dans $M$ dont l'image est une $k$-surface et telle que $i$ est l'application qui à un point de $S$ associe l'extrémité à l'infini de la normale extérieure à $f(S)$ en ce point.

La terminologie se justifie par le fait que lorsque $i$ est un plongement du disque ouvert qui s'étend à un plongement du disque fermé, alors on a envie de penser que le bord à l'infini de la solution coincïde avec le bord du disque. Elle peut-être aussi source de confusion car en général une solution d'un problème asymptotique n'a pas de bord à l'infini en un sens raisonnable.

Nous démontrons une série de résultats sur ce problème de Plateau asymptotique.

Théorème A. Il existe au plus une solution du problème de Plateau asymptotique. Le problème de Plateau asymptotique n'a pas toujours de solutions :

Théorème B. Si $S$ est $\partial_{\infty} M$ auquel on a ôté 0,1 , ou 2 points et si $i$ est l'injection canonique, alors le problème $(i, S)$ n'a pas de solutions.

Nous avons les résultats d'existence suivants : 
Théorème C. Si $(i, S)$ est un problème de Plateau asymptotique et si $\partial_{\infty} M \backslash i(S)$ contient au moins trois points distincts, alors $(i, S)$ admet une solution.

Théorème D. Si $\Gamma$ est un groupe agissant sur $S$, tel que $S / \Gamma$ soit une surface compacte de genre plus grand ou égal à 2 , si $\rho$ est une représentation de $\Gamma$ dans le groupe des isométries de $M$, et $i$ vérifie

$$
\forall \gamma \in \Gamma, \quad i \circ \gamma=\rho(\gamma) \circ i
$$

alors le problème $(i, S)$ a une solution.

Enfin

Théorème E. Si $(i, U)$ est un problème de Plateau asymptotique et si $S$ est un ouvert relativement compact de $U$ alors $(i, S)$ admet une solution.

On peut remarquer à ce stade une analogie entre les données de problèmes de Plateau asymptotiques ayant des solutions, et les immersions hyperboliques du disque dans $\mathbb{C} P^{1}$.

Nous avions démontré dans [4] le théorème $\mathrm{D}$ dans le cas où la variété ambiante était à courbure constante. Toujours dans le cas de la courbure ambiante contante, mais en toutes dimensions, J. Spruck et H. Rosenberg ont démontré des versions partielles de nos résultats : ils ont montré que le problème de Plateau défini par $(i, U)$ avait une solution dans le cas où $U$ était l'intérieur du disque fermé $D$ et où $i$ s'étendait en un plongement de $D$ dans $U$ [5]. Les autres résultats de cet article sont des cas particuliers, toujours dans le cadre de la courbure ambiante constante ( mais en toutes dimensions ), de 5.0.3 : les auteurs ne considèrent que les graphes au dessus d'une horosphère. Ceci leur permet d'écrire explicitement les équations satisfaites par la fonction dont une telle surface est le graphe, équations qui, grâce à l'hypothèse de coubure ambiante constante, sont d'une forme simple à laquelle peuvent s'appliquer les estimées a priori, classiques pour les équations de Monge-Ampère. Quant à nous, nous appliquons les techniques plus flexibles de courbes pseudo-holomorphes mises au point dans [3]. Ceci nous permet de soritir du cadre de la courbure constant, et de considérer des surfaces qui ne sont pas des graphes au dessus des horosphères.

\subsection{Propriétés hyperboliques de l'espace des $k$-surfaces}

Nous voulons poursuivre notre analogie avec les géodésiques plus loin. Soit maintenant $N$ une variété de dimension 3 compacte. Le fibré unitaire de $N$ est l'espace des géodésiques pointées, c'est-à-dire des paires $(\gamma, x)$ où $x$ est un point de la géodésique $\gamma$. Le flot géodésique correspond alors au feuilletage de dimension 1 obtenu en faisant bouger le point le long d'une géodésique donnée. 
Définissons donc les $k$-surfaces pointées, comme les paires $(S, x)$ où $x$ est un point sur la $k$-surface $S$. Nous attirons encore une fois l'attention sur le fait qu'il s'agit de surfaces en général non compactes et ayant même beaucoup de récurrence. Nous définissons également les tubes pointés comme les paires $(T, x)$, où $T$ est une géodésique et $x$ est un vecteur normal unitaire à $T$.

Dans [3], dans le cadre plus général des problèmes de Monge-Ampère, nous avons montré l'espace $\mathcal{N}$, constitué des $k$-surfaces pointées et des tubes pointés, est compact, et possède de plus une structure de lamination définie par la condition : deux points $(F, x)$ et $(G, y)$ sont sur la même feuille si $F=G$.

Par abus le langage, nous appelerons $\mathcal{N}$ l'espace des $k$-surfaces de $N$.

Nous donnerons plus de précisions sur la topologie sous-jacente dans 8 et renvoyons surtout à [3].

Le résultat essentiel de notre article est le théorème suivant qui montre que l'espace $\mathcal{N}$ est une bonne généralisation du flot géodésique, car il en possède les propriétés hyperboliques.

Théorème F. Soit $\mathcal{N}$ l'espace des $k$-surfaces d'une variété compacte $N$ munie d'une métrique $g$ à courbure plus petite que -1, alors

(i) pour tout entier $g$, l'ensemble des feuilles compactes de genre plus grand que $g$ est dense dans $\mathcal{N}$,

(ii) une feuille générique est dense dans $\mathcal{N}$,

(iii) enfin $\mathcal{N}$ est stable dans le sens suivant, si $h$ est une métrique suffisamment proche de $g$, et si $\overline{\mathcal{N}}$ est l'espace des $k$-surfaces pour la métrique $h$, alors il existe un homéomorphisme entre $\mathcal{N}$ et $\overline{\mathcal{N}}$ envoyant feuille sur feuille

L'espace des $k$-surfaces contient l'espace des tubes pointés, espace qui fibre au-dessus du flot géodésique. En ce sens, l'espace des $k$-surfaces "contient le flot géodésique". Dans le cas, où la courbure ambiante est constante, il contient également l'espace des plans totalement géodésiques $\mathcal{P}$ : les surfaces équidistantes des plans totalement géodésiques sont à courbure constante. Notre espace $\mathcal{N}$ de dimension infinie est cependant beaucoup plus gros.

Signalons ici à titre de comparaison quelques propriétés de l'espace $\mathcal{P}$ des plans totalement géodésiques toujours dans le cas de la courbure ambiante constante. Tout d'abord, par le théorème d'ergodicité de Moore, une feuille générique est dense. En ce qui concerne les feuilles compactes, il est connu que pour certaines variétés arithmétiques $\mathcal{P}$ ne contient aucune feuilles compactes, pour d'autres il en contient un ensemble dense. 
Cet espace $\mathcal{P}$ des plans totalement géodésiques, n'a bien sûr pas beaucoup d'intérêt quand la courbure n'est pas constante, mais il possède une sorte de propriété de "stabilité" démontrée par M. Gromov [2] : si une métrique $h$ sur une variété $N$ est suffisamment proche d'une métrique $g$ à courbure constante, alors il existe une application continue de $\mathcal{P}$, l'espace des plans totalement géodésiques pour $g$, dans $N$ telle que les images des feuilles soient des surfaces minimales.

La situation décrite par le théorème $\mathrm{F}$ amène à se poser un certain nombre de questions :

(i) on a une abondance de mesures tranverses invariantes, mais y en a-t-il une qui charge tous les ouverts?

(ii) Quelle est la statistique des feuilles compactes? On peut associer à chaque $k$-surface l'intégrale de sa courbure moyenne, pour de bonnes raisons nous appelerons ceci l'aire de la $k$-surface. De manière naturelle, l'aire des tubes est alors la longueur de la géodésique sous-jacente ( à $2 \pi$ près ). On peut alors montrer ( ce n'est pas fait dans cet article ) qu'il n'y a qu'un nombre fini $N(A)$ de feuilles compactes d'aire bornée par $A$. Notons $N(A, g)$ le nombre de celles-ci qui sont de genre $g$. On a envie de poser

$$
\operatorname{ent}_{g}(N)=\liminf _{h \leq g,} \frac{\log (N(A, g))}{A} .
$$

Le nombre $e n t_{0}$ est l'entropie du flot géodésique, quelles sont les valeurs de ent $t_{g}$ ?

(iii) Les feuilles compactes sont elles équidistribuées?

(iv) On peut également se poser une question qui est l'analogue de la question de conjugaison pour les flots géodésiques : pour deux métriques proches, supposons que l'homéomorphisme de conjugaison puisse être choisi conforme sur chacune des feuilles, les métriques sont-elles isométriques?

\subsection{Structure de l'article}

2- Définitions, énoncé du lemme de Morse. Nous énonçons le lemme de Morse, ainsi que les définitions nécessaires. Cette section contient également des définitions utilisées de manière récurrente dans l'article ainsi que quelques propriétés préliminaires.

3 - Variations infinitésimales et déformations. Nous y démontrons le lemme 5.3.1 qui décrit les variations infinitésimales de $k$-surfaces à bord.

4- Théorème de compacité. Nous y étudions les limites de $k$-surfaces. Cette section débute par un rappel des résultats de compacité sur les problèmes de Monge-Ampère démontrés dans [3]. 
5 - Problème de Plateau pour les disques. Nous y démontrons la proposition 5.0 .3 qui est une version faible du lemme de Morse.

6- Démonstration du lemme de Morse pour les surfaces convexes. Nous démontrons l'existence et l'unicité, en utilisant une méthode de déformation.

7- Problèmes asymptotiques. Nous y démontrons les théorèmes énoncés dans l'introduction. Nous traitons le théorème $\mathrm{A}$ en 7.2.1; les théorèmes $\mathrm{C}, \mathrm{D}$ et $\mathrm{E}$ sont vus

en 7.3.3, 7.3.1 et 7.3 .2 respectivement, et enfin $\mathrm{B}$ est démontré en 7.4 .1 .

8- Espace des $k$-surfaces. A partir de cette section, nous nous intéressons à l'espace des $k$-surfaces (sans bord) d'une variété compacte. Cet espace est introduit en 5.3.1. 9- Densité des feuilles périodiques. Il s'agit de de F-(i). La démonstration est la plus délicate de cet article.

10-Généricité des feuilles denses. Nous y démontrons F-(ii).

11-Stabilité. Cette section contient la preuve de F-(iii).

\section{Définitions}

Dans cette section, nous allons présenter les définitions utilisées dans l'énoncé du lemme de Morse. Nous donnerons également quelques définitions connexes utilisées dans les preuves, ainsi que quelques résultats préliminaires.

Enonçons tout le suite le lemme de Morse qui sera démontré en 6.0 .2

Lemme de Morse. Soit $M$ une variété d'Hadamard à géométrie bornée et à courbure strictement plus petite que $-c<0$. Soit $S$ une surface localement convexe, éventuellement à bord, à courbure plus grande que $c$, à géométrie bornée et qui n'est ni horosphérique à l'infini, ni tubulaire, ni compacte sans bord. Alors, pour tout $k \in] 0, c[$, il existe une unique $k$-surface lentille pour $S$.

De plus, si $S$ n'est pas tubulaire à l'infini, cette $k$-surface a sa courbure moyenne uniformément bornée et en particulier n'est pas dégénérée

Les termes de ce lemme vont être expliqués dans cette section.

Nous parlerons des $k$-surfaces dégénérées et non dégénérées dans le paragraphe 2.2. Nous définirons les surfaces lentilles en 2.2.2 et nous aurons besoin de 2.2.1 pour cela. Le paragraphe sur la géométrie bornée 2.3 est nécessaire pour donner la définition 2.4 de tubulaire, horosphérique à l'infini etc ....

Cette section contient également des résultats souvent utilisés cet article. 


\section{$2.1 \quad k$-surfaces}

Si $S$ est une surface convexe immergée dans une variété de dimension $3 \mathrm{M}$, nous noterons $n(S)$ son relevé de Gauss, c'est-à-dire la surface immergée dans le fibré unitaire $U M$ de $M$ constituée des vecteurs normaux extérieurs à $S$.

Une $k$-surface est une surface (éventuellement à bord) immergée dans $M$ dont le produit des courbures principales vaut $k$, et telle que la métrique induite de $n(S)$ soit complète. Attention, ceci n'entraîne pas a priori que la métrique induite de celle de $M$ est complète. Si tel est le cas, la surface est non dégénérée, et dégénérée dans le cas contraire. Si la courbure moyenne est bornée, alors la $k$-surface est non dégénérée.

\subsection{Normal étendu, surfaces lentilles, bout}

\subsubsection{Normal étendu, bout}

Soit $S$ une surface localement convexe immergée à bord $\partial S$ dans $M$. Soit $n$ son champ de vecteur normal extérieur et $n_{\partial}$ le champ de vecteur normal intérieur à $\partial S$ dans $S$. Posons

$$
N_{\partial}^{+}=\left\{u \in(T \partial S)^{\perp} /\left\langle u, n_{\partial}\right\rangle \leq 0\right\} .
$$

Le normal étendu de $S$ est le sous-ensemble $N_{S}$ de $U M$, défini par

$$
N_{S}=n(S) \cup N_{\partial}^{+}
$$

Dans la suite, nous identifierons souvent abusivement $n(S)$ ( vu comme sousensemble de $U M$ ) et $S$. Il est facile de voir que $N_{S}$ est une sous-variété $C^{0}$-immergée dans $U M$ dont le bord $\partial N_{S}$ est l'ensemble de vecteurs normaux intérieurs à $S$ le long de $\partial S$.

Le bout $B$ de $S$ sera la variété de dimension 3 , homéomorphe à $\left.N_{S} \times\right] 0,+\infty$ [ et muni de la métrique induite par l'application

$$
\left\{\begin{array}{c}
\left.N_{S} \times\right] 0,+\infty[\rightarrow M \\
(t, n) \mapsto \exp (t n)
\end{array}\right.
$$

D’un point de vue métrique, on compléte le bout en ajoutant $S$, on parlera alors d'un bout complet. 


\subsubsection{Graphe étendu, surface lentille, champ focal, pied, fonctions in- verses}

Une surface $\Sigma$ localement convexe immergée à bord $\partial \Sigma$ est un graphe étendu au-dessus de $S$ s'il existe

- un ouvert $U$ de $\operatorname{int}\left(N_{S}\right)=N_{S} \backslash \partial N_{S}$, vérifiant

$$
n \overline{(S)} \subset U \subset \bar{U} \subset \operatorname{int}\left(N_{S}\right)
$$

où $\bar{A}$ désigne l'adhérence de $A$ dans $N_{S}$,

- une fonction $f$ continue définie sur $\bar{U}$, strictement positive sur $U$, tels que

(i) $\forall y \in \bar{U} \backslash U, \quad f(y)=0$,

(ii) $\Sigma=\{\exp (f(u) u) / u \in U\}$,

(iii) le segment géodésique $\exp ([f(u),+\infty[u)$ est extérieur à $\Sigma$.

Enfin, si $\Sigma$ est le graphe étendu pour une fonction $f$ au-dessus de $S$, nous dirons que $\Sigma$ est un graphe étendu borné au-dessus de $S$ si $f$ est bornée et de manière symétrique que $S$ est lentille pour $\Sigma$, et nous appellerons $f$ la fonction associée.

Le champ de vecteur focal pour un graphe étendu $\Sigma$ au-dessus de $S$ sera le champ de vecteur

$$
U:\left.\exp (f(u) u) \mapsto \frac{d}{d t}\right|_{t=f(u)} \exp (t u) .
$$

Enfin si $\Sigma$ est un graphe étendu au-dessus de $S$, le pied d'un point $y=$ $\exp (f(u) u)$ de $\Sigma$ sera le point $x=\pi(u)$ de $S$, où $\pi$ est la projection du fibré unitaire de $M$ sur $M$.

\subsubsection{Remarques}

(i) L'ouvert $U$ de la définition d'un graphe étendu contenant l'adhérence de $n(S)$ et $f$ étant strictement positive, $\nu(s) \neq n(s)$ pour tous les points $s$ de $\partial S$, où $\nu$ est le champ de vecteur normal extérieur à $\Sigma$.

(ii) Soit $\Sigma$ est un graphe étendu au-dessus de $S$. Si $\nu$ est le champ de vecteur normal extérieur à $\Sigma$ alors $\nu(\partial S) \subset \operatorname{int}\left(N_{S}\right)$. En effet, d'après 2.2 .2 (iii), le vecteur $n_{\partial}$ pointe vers l'intérieur de $\Sigma$, c'est-à-dire, si $s$ est un point de $\partial S=\partial \Sigma$, nous avons

$$
\left\langle n_{\partial}(s), \nu(s)\right\rangle \leq 0 .
$$


Ensuite, $\nu(s)$ étant perpendiculaire à $T(\partial S)$ appartient au plan engendré par $n_{\partial}(s)$ et $n(s)$. En particulier, si $\nu(s) \notin \operatorname{int}\left(N_{S}\right)$, nous avons nécessairement $\nu(S)=-n(S)$, mais ceci est impossible : deux surfaces strictement convexes ayant un point d'intersection et en ce point deux normales opposées sont telles que, au moins localement, leur intersection est réduite à ce point.

(iii) Un graphe étendu $\Sigma$ au-dessus de $S$, se plonge naturellement dans le bout complet de $S$ de telle sorte que $\partial \Sigma \subset \partial S$.

\subsubsection{Fonction inverse}

Introduisons une denière notion utile. Si $S$ est une surface lentille pour $\Sigma$ de fonction associée $\lambda$, la fonction inverse est la fonction $\mu$ définie sur $\Sigma$ par

$$
\mu(\exp (\lambda(u) u))=\lambda(u)
$$

Nous avons alors le

\section{Lemme 2.2.1 Toute fonction inverse est 2-lipschitzienne}

Preuve :notons $\mu$ la fonction inverse. Soient $u$ et $v$ deux éléments de $N_{S}$. En notant $x=\pi(u)$ et $y=\pi(v)$, où $\pi$ est la projection de $U M$ sur $M, d_{\Sigma}$ la distance riemanienne intrinséque de $\Sigma$, nous avons par convexité locale de $S$ et grâce à notre hypothèse de courbure négative

$$
d(x, y) \leq d_{\Sigma}(\exp (\lambda(u) u), \exp (\lambda(v) v)
$$

Enfin comme

$$
|\lambda(u)-\lambda(v)| \leq d(x, y)+d_{\Sigma}(\exp (\lambda(u) u), \exp (\lambda(v) v),
$$

Nous en déduisons bien le résultat.»

\subsubsection{Déformations}

Grâce à la remarque 2.2.3 (i), nous avons le lemme évident de déformation suivant

Lemme 2.2.2 Soit $S_{t}, t \in[0,1]$ une famille de surfaces immergées localement convevoi morse.dvi xes, compactes à bord. Soit $\Sigma_{t}$ une autre famille de surfaces compactes, localement convexes, immergées et telle que $\partial S_{t}=\partial \Sigma_{t}$. Alors, si $\Sigma_{0}$ est lentille pour $S_{0}$ alors $\Sigma_{t}$ est lentille pour $S_{t}$ pour tout $t$ dans un voisinage de 0. 


\subsection{Géométrie bornée}

Rappelons quelques définitions.

\subsubsection{Convergence de variétés pointées}

Une variété pointée est une paire $(M, x)$ où $M$ est une variété et $x$ un point de $M$. Nous dirons que la suite de variétés pointées $\left\{\left(M_{n}, x_{n}\right)\right\}_{n \in \mathbb{N}}$ équipées des métriques $g_{n}$ converge $C^{\infty}$ sur tout compact vers la variété riemanienne $\left(M_{\infty}, x_{\infty}\right)$ équipée de la métrique $g_{\infty}$ s'il existe une suite d'applications $\left\{f_{n}\right\}_{n \in \mathbb{N}}$ (pas nécessairement continues) définies de $M_{\infty}$ dans $M_{n}$, envoyant $x_{\infty}$ dans $x_{n}$ telle que pour tout $R$, alors

(i) pour $n$ suffisamment grand, la restriction de $f_{n}$ à la boule de rayon $R$ et de centre $x_{\infty}$ est $C^{\infty}$ et injective

(ii) $f_{n}^{*} g_{n}$ converge $C^{\infty}$ sur tout compact vers $g_{\infty}$.

Nous dirons qu'une variété $M$ est à géométrie bornée si pour toute suite de points $\left\{x_{n}\right\}_{n \in \mathbb{N}}$ de $M$, la suite de variétés pointées $\left\{\left(M, x_{n}\right)\right\}_{n \in \mathbb{N}}$ possède une sous-suite convergente.

Par abus de langage, nous dirons qu'une variété $M$ d'Hadamard est à géométrie bornée si quelque soit la suite de points $\left\{x_{n}\right\}_{n \in \mathbb{N}}$ de $M$, la suite de variétés pointées $\left\{\left(M, x_{n}\right)\right\}_{n \in \mathbb{N}}$ possède une sous-suite convergente vers une variété à courbure strictement négative.

\subsubsection{Remarques}

(i) Une variété de Hadamard ayant un groupe discret cocompact d'isométries est à géométrie bornée.

(ii) Si une variété de Hadamard à courbure négative est à géométrie bornée, alors la croissance des horosphères est polynomiale. Remarquons en effet tout d'abord que la courbure reste coincée entre deux bornes strictement négatives. Le flot géodésique de $M$ va donc être d'Anosov. De plus, par géométrie bornée le volume des boules de rayon 1 sur les horosphères va être uniformément borné. L'argument classique montrant la croissance polynomiale des variétés stables d'un flot d'Anosov nous fournit le résultat. 


\subsubsection{Convergence de sous-variétés immergées}

Nous nous intéresserons aux variétés immergées pointées, c'est-à-dire aux quadruplets de la forme $Q=(N, x, f, M)$ où $x$ est un point d'une variété $N, f$ une immersion de $N$ dans une variété riemanienne $M$. Nous dirons qu'une suite de variétés immergées $\left\{Q_{n}=\left(N_{n}, x_{n}, f_{n}, M_{n}\right)\right\}_{n \in \mathbb{N}}$ converge $C^{\infty}$ sur tout compact vers une variété immergée $Q_{\infty}=\left(N_{\infty}, x_{\infty}, f_{\infty}, M_{\infty}\right)$ si

(i) La suite de variétés pointées $\left\{\left(M_{n}, f_{n}\left(x_{n}\right)\right)\right\}_{n \in \mathbb{N}}$ converge vers la variété pointée $\left(M_{\infty}, f_{\infty}\left(x_{\infty}\right)\right)$

(ii) La suite de variétés pointées $\left\{\left(N_{n}, x_{n}\right)\right\}_{n \in \mathbb{N}}$, où $N_{n}$ est munie de la métrique induite par $f_{n}$, converge vers $\left\{\left(N_{\infty}, x_{\infty}\right)\right\}$, où $N_{\infty}$ est munie de la métrique induite par $f_{\infty}$.

(ii) $\left\{f_{n}\right\}_{n \in \mathbb{N}}$ converge vers $f_{\infty}$ sur tout compact au sens où on l'imagine.

Enfin, une variété immergée $(N, x, f, M)$ sera dite à géométrie bornée si pour toute suite $\left\{x_{n}\right\}_{n \in \mathbb{N}}$ la suite $\left\{\left(N, x_{n}, f, M\right)\right\}_{n \in \mathbb{N}}$ admet une sous-suite convergente.

Plus généralement nous dirons qu'une suite de variétés immergées

$$
\left\{\left(N_{n}, x_{n}, f_{n}, M_{n}\right)\right\}_{n \in \mathbb{N}}
$$

est à géométrie bornée si pour toute suite de points $\left\{y_{n} \in N_{n}\right\}_{n \in \mathbb{N}}$, la suite

$$
\left\{\left(N_{n}, y_{n}, f_{n}, M_{n}\right)\right\}_{n \in \mathbb{N}}
$$

possède une sous-suite convergente.

Lorsque la variété ambiante $M$ est sous-entendue, nous abrévierons souvent le quadruplet $(N, x, f, M)$ décrivant une variété immergée, en une paire $(N, x)$ et en confondant ainsi de manière abusive $N$ et son image.

\subsection{Surfaces horosphériques, surface tubulaires}

Dans ce paragraphe, $M$ sera toujours une variété simplement connexe de dimension 3 à courbure strictement négative et à géométrie bornée.

\subsubsection{Surfaces horosphériques, pseudo-horosphères}

Par définition, une surface horosphérique sera une surface convexe complète plongée $\Sigma$, pour laquelle il existe une fonction de Busemann $h$ vérifiant les deux conditions suivantes 
(i) $h$ est majorée sur $\Sigma$,

(ii) le gradient de $h$ est partout transverse à $\Sigma$.

Une suite de surfaces immergées $\left\{\left(S_{n}, x_{n}\right)\right\}_{n \in \mathbb{N}}$ sera dite de type horosphérique s'il existe une suite de points $\left\{y_{n} \in S_{n}\right\}_{n \in \mathbb{N}}$ telle que $\left\{\left(S_{n}, y_{n}\right)\right\}_{n \in \mathbb{N}}$ possède une soussuite qui converge vers une surface horopshérique.

Une surface immergée $(\Sigma, x)$ sera dite horosphérique à l'infini s'il existe une suite de points $\left\{x_{n}\right\}_{n \in \mathbb{N}}$ de $\Sigma$ telle que $\left\{\left(\Sigma, x_{n}\right)\right\}_{n \in \mathbb{N}}$ converge vers une surface horosphérique.

Nous dirons qu'une surface convexe complète plongée $S$ dans $M$ est une pseudo-horosphère si l'application de Gauss-Minkowski définie de $S$ dans $\partial_{\infty} M$ par,

$$
x \mapsto \exp (+\infty n(x))
$$

où $n$ est le champ de vecteur normal extérieur, est une bijection sur $\partial_{\infty} M$ privé d'un point.

Une surface horosphérique est une pseudo-horosphère et réciproquement nous avons le

Lemme 2.4.1 Dans une variété à géométrie bornée une pseudo-horosphère à géométrie bornée est horosphérique à l'infini

Preuve :soit $y \in \partial_{\infty} M$ le point évité par l'application de Gauss-Minkowski de la pseudo-horosphère $S$. Soit $B$ l'ensemble convexe bordé par $S$. Construisons une suite de convexes $\left\{C_{n}\right\}_{n \in \mathbb{N}}$ ayant les propriétés suivantes

(i) $\partial_{\infty} C_{n}$ est un voisinage de $y$,

(ii) $\left\{C_{n}\right\}_{n \in \mathbb{N}}$ converge au sens de Haussdorff dans le compactifié de $M$ vers

$y$.

Soit maintenant $d_{n}$ la fonction distance à $C_{n} \cap B$. D'après (i) sur $S, d_{n}$ atteint son maximum en un point $x_{n}$. D'après (ii), $d_{n}\left(x_{n}\right)$ tend vers $+\infty$.

Considérons la suite de surfaces immergées $\left\{\left(S, x_{n}\right)\right\}_{n \in \mathbb{N}}$ immergées dans la suite $\left\{\left(M, x_{n}\right)\right\}_{n \in \mathbb{N}}$. Par nos hypothèses, $\left\{\left(S, x_{n}\right)\right\}_{n \in \mathbb{N}}$ va converger vers une surface $\left(S_{\infty}, x_{\infty}\right)$ immergée dans une variété $\left(M_{\infty}, x_{\infty}\right)$. Enfin la suite de fonctions $d_{n}-d_{n}\left(x_{n}\right)$ va converger vers une fonction de Buseman sur $M_{\infty}$, majorée sur $S$ et dont le gradient est dirigé vers l'extérieur de $S_{\infty}$. La surface $S_{\infty}$ est donc de type horosphérique, et $S$ est donc bien horosphérique à l'infini.» 


\subsubsection{Surfaces tubulaires, tubes}

Par définition, une surface tubulaire sera une surface localement convexe complète plongée $\Sigma$, pour laquelle il existe une géodésique telle que si $d$ est la fonction distance à cette géodésique, $d$ vérifie les deux conditions suivantes

(i) $d$ est bornée sur $\Sigma$,

(ii) le gradient $u$ de $d$ est dirigé vers l'extérieur de $\Sigma$, c'est-à-dire $\langle u, n\rangle \geq 0$, où $n$ est le champ de vecteur normal extérieur.

Il est intéressant de remarquer que la surface étant convexe, et les lignes de gradient de $d$ étant des géodésiques, la condition (iii) est équivalente à la condition où on impose $\langle u, n\rangle>0$.

Une suite de surface immergée sera dite de type tubulaire si elle possède une sous-suite qui converge vers une surface tubulaire.

Une surface immergée $(\Sigma, x)$ sera dite tubulaire à l'infini s'il existe une suite de points $\left\{x_{n}\right\}_{n \in \mathbb{N}}$ de $\Sigma$ telle que $\left\{\left(\Sigma, x_{n}\right)\right\}_{n \in \mathbb{N}}$ converge vers une surface tubulaire.

Enfin une définition connexe nous sera souvent utile par la suite : le tube d'une géodésique $\gamma$ est défini par

$$
N(\gamma)=\{u \in U M /\langle u, \dot{\gamma}\rangle=0\}
$$

\subsubsection{Remarque}

Il est utile de remarquer qu'être de type horosphérique, tubulaire, horosphérique ou tubulaire à l'infini est une propriété qui ne dépend que de $\left\{S_{n}\right\}_{n \in \mathbb{N}}$ et $S$ réciproquement et ne fait pas intervenir les points choisis sur ces surfaces

\subsection{Principe du maximum géométrique et applications}

Nous avons l'énoncé évident suivant que nous appelerons du nom pompeux de principe du maximum géométrique.

Lemme 2.5.1 Soit $S_{1}$ et $S_{2}$ deux surfaces convexes tangentes en un point $x$ et telle que $S_{1}$ est à l'intérieur de $S_{2}$ au voisinage de $x$, alors $k_{1}(x) \geq k_{2}(x)$, où $k_{i}$ désigne la courbure extrinsèque de $S_{i}$.

Nous allons en tirer quelques conséquences. Pour cela supposons que la courbure de $M$ est plus petite que $-c<0$ et soit $k$ tel $0<k<c$. Nous avons

Proposition 2.5.2 Si $S$ est une $k$-surface compacte telle que $\partial S$ est inclus dans une boule $B$, alors $S$ toute entière est incluse dans cette boule. 
Preuve :en effet avec nos hypothèses, toute sphère a une courbure plus grande que $c$.

Rappelons en rapidement la démonstration en utilisant 3.1.1: si nous notons $\lambda_{i}^{r}$ les valeurs propres de l'opérateur deuxième forme fondamentale de la sphère de rayon $r, S^{r}$, associées aux vecteurs propres $e_{i}^{r}$, ainsi que $k_{i}^{r}$ les courbures sectionelles des plans perpendiculaires à $S^{r}$ passant par $e^{i}$, d'après 3.2.1, la courbure $\kappa_{r}$ de la sphère de rayon $r$ vérifie l'équation

$$
\frac{d \kappa_{r}}{d r}=-\kappa_{r}\left(\lambda_{2}^{r}\left(1+\frac{k_{1}^{r}}{\kappa_{r}}\right)+\lambda_{1}^{r}\left(1+\frac{k_{2}^{r}}{\kappa_{r}}\right)\right)
$$

La propriété annoncée découle immédiatement de l'inégalité :

$$
\frac{d \kappa_{r}}{d r} \geq\left(\kappa_{r}-c\right)\left(-\lambda_{1}^{r}-\lambda_{2}^{r}\right) .
$$

Nous pouvons conclure par l'absurde maintenant. Si $S$ n'est pas incluse dans $B$, on peut construire une sphère à laquelle $S$ est tangente intérieurement ce qui contredit notre principe du maximum. $\diamond$

Le même raisonnement montre

Proposition 2.5.3 Une k-surface ne peut-être horosphérique.

\subsection{Domination}

Soit $S$ une surface lentille pour $\Sigma$, de fonction associée $f$ définie sur l'adhérence d'un ouvert $U$ du normal étendu $N_{S}$ à $S$. Nous dirons que $S$ domine la surface $S_{1}$, s'il existe un ouvert $V$ de $U$ tel que $\bar{V} \subset U$, où $\bar{V}$ est l'adhérence de $V$ dans $N_{S}$, une fonction positive ou nulle $g$ définie sur $\bar{V}$, inférieure ou égale à $f$ sur $V$ et égale à $f$ sur $\bar{V} \backslash V$, telle que $S_{1}$ est le graphe de $g$ sur $V$. Dans le cas où la fonction $g$ est strictement positive, nous dirons que $S$ domine strictement la surface $S_{1}$

Un lemme facile et utile est le

Lemme 2.6.1 Soient $S$ une surface lentille pour $\Sigma, \Sigma_{t}$ une famille d'ouverts à bord de $\Sigma$ pour $t \in[0,1]$ et $S_{t}$ une famille de surfaces lentilles pour $\Sigma_{t}$, alors

(i) l'ensemble des $t$ tels que $S$ domine strictement $S_{t}$ est ouvert,

(ii) si $\forall t \in] 0,1], S$ domine strictement $S_{t}$ alors, soit $S$ domine strictement $S_{0}$, soit $S$ est tangente intérieurement à $S_{0}$ en un point intérieur à $S$. 


\section{Variations infinitésimales et déformations}

Le but essentiel de cette section est le

Lemme 3.0.2 Soit $k$ un réel positif tel que $M$ soit à courbure strictement plus petite que $-k$. Soit $\Sigma$ une $k$-surface compacte à bord $\partial \Sigma$ et soit $c_{t}$, une famille continue de déformations de $\partial \Sigma$ telle que $c_{0}=\partial \Sigma$, soit $k(t)$ une fonction $C^{\infty}$ de $t$ telle que $k(0)=k$. Il existe alors une famille unique de $k(t)$-surfaces immergées $\Sigma^{t}$, définie au voisinage de 0 , telle que $\Sigma^{0}=\Sigma$ et $\partial \Sigma^{t}=c^{t}$.

De plus, s'il existe une famille immergée de surfaces $S^{t}$ vérifiant $c_{t}=\partial S^{t}$, telle que $\Sigma$ est lentille pour $S^{0}$, alors $\Sigma^{t}$ est lentille pour $S^{t}$ au voisinage de 0.

Enfin, si $S^{t} \subset S^{s}$ pour $t \geq s$ et $k(t)$ est croissante alors $\Sigma$ domine $\Sigma^{t}$, toujours pour $t$ au voisinage de 0 . Si de plus $S^{t} \neq S^{0}$ ou $k(t)$ est strictement croissante, alors $\Sigma$ domine strictement $\Sigma^{t}$ pour $t$ au voisinage de zéro.

Le coeur de la démonstration de cette proposition est une proposition de déformation infinitésimale que nous allons maintenant présenter.

Soit $S \subset M$ une surface compacte immergée à bord. Notons $C_{0}^{\infty}(S)$ l'espace des fonctions $C^{\infty}$ définies sur $S$ et nulles sur le bord.

A toute $f \in C^{\infty}(S)$, on peut associer une variation de surfaces définie pour $t$ suffisamment petit par les immersions $s_{t}^{f}$

$$
\begin{gathered}
S \\
x \\
\longrightarrow \exp (\operatorname{tfn}(x))=s_{t}^{f}(x)
\end{gathered}
$$

où $n$ désigne le champ de vecteur normal extérieur à $S$.

Désignons par $k_{t}^{f}(x)$ la courbure extrinsèque à la surface $s_{t}^{f}(S)$ au point $s_{t}^{f}(x)$, l'opérateur $L$ de variation infinitésimale de courbure extrinsèque est alors

$$
\begin{aligned}
C_{0}^{\infty}(S) & \longrightarrow C^{\infty}(S) \\
f & \longmapsto L(f)=\left.\frac{d}{d t}\right|_{t=0} k_{t}^{f} .
\end{aligned}
$$

Nous montrerons

Proposition 3.0.3 Soit $k$ un réel positif. Si $M$ est à courbure strictement plus petite que $-k$ et $S$ est à courbure extrinsèque strictement comprise entre 0 et $k$, alors l'opérateur $L$ est elliptique et inversible

Dans cette section, nous expliciterons tout d'abord l'opérateur $L$, puis démontrerons 3.0 .3 en utilisant le principe du maximum. Enfin nous montrerons 3.0.2 


\subsection{Explicitation de l'opérateur $L$}

Nous allons calculer explicitement l'opérateur $L$ pour une surface quelconque $S$. Notons pour cela $\nu$ la connexion de Levi-Civita de $M, R$ son tenseur de courbure, $n$ le vecteur normal à $S, W$ l'endomorphisme de $T S$ défini par $W(u)=R(n, u) n$, $\kappa$ la courbure extrinsèque de $S, B$ l'opérateur deuxième forme fondamentale de $S$ défini par $B(u)=\nu_{u} n$ et enfin $\operatorname{Hess}(f)$ la hessienne de $f$.

Montrons alors

Proposition 3.1.1 Nous avons

$$
L(f)=\kappa\left(-\operatorname{trace}\left(\operatorname{Hess}(f) \circ B^{-1}\right)+f \operatorname{trace}\left(W \circ B^{-1}\right)-f \operatorname{trace}(B)\right)
$$

Preuve :donnons tout d'abord le cadre de ce calcul. Il nous faut considérer la famille d'immersions $s_{t}^{f}$ comme une application $s^{f}$ de $S \times \mathbb{R}$ dans $M$. Nous identifions $S$ à $S \times\{0\}$. Sur le fibré $E$ induit de $T M$ par $s^{f}$, nous noterons par abus de langage $\nu$ la connexion induite de la connexion de Levi-Civita de $M$. Nous pouvons maintenant voir $T s^{f}=F$, comme une section de $T S^{*} \otimes E$, et nous avons bien sûr $d^{\nu} F=0$ et $F\left(\frac{\partial}{\partial t}\right)=f n$ le long de $S$.

Tout champ de vecteur $u$ sur $S$ donne canoniquement naissance à un champ de vecteur noté également $u$ sur $S \times \mathbb{R}$ qui commute avec le champ $\frac{\partial}{\partial t}$. Considérons également $n_{t}$ le champ de vecteur normal à $S_{t}=s_{t}^{f}(S)$, et $n$ la section de $E$ qui s'en déduit.

Par abus de notation, si $v$ est une section de ce fibré $E$ nous noterons

$$
\left.\frac{d}{d t}\right|_{t=0} v(x)=\left(\nu_{\frac{\partial}{\partial t}} v\right)(x, 0)
$$

Considérons $A$, la section de $T S^{*} \otimes E$ définie par

$$
A(u)=\nu_{u} n
$$

Un premier calcul donne

$$
\left.\frac{d}{d t}\right|_{t=0} F(u)=\nu_{u}(f n)=d f(u) \cdot n+f A(u) .
$$

En notant $\langle$,$\rangle la métrique de M$ et celle qui s'en déduit sur $E$, nous avons

$$
0=\left.\frac{d}{d t}\right|_{t=0}\langle n, F(u)\rangle=\left\langle\left.\frac{d}{d t}\right|_{t=0} n, F(u)\right\rangle+\left\langle n,\left.\frac{d}{d t}\right|_{t=0} F(u)\right\rangle .
$$


Nous en tirons facilement

$$
\left.\frac{d}{d t}\right|_{t=0}=F(-\nu f)
$$

Nous obtenons ainsi

$$
\begin{aligned}
\left.\frac{d}{d t}\right|_{t=0} A(u) & =\nu_{\frac{\partial}{\partial t}} \nu_{u} n \\
= & R\left(\frac{\partial}{\partial t}, u\right) n+\nu_{u} \nu_{\frac{\partial}{\partial t}} n \\
= & f R(n, u) n-\nu_{u} \nu f .
\end{aligned}
$$

Considérons $g$ la métrique induite sur $T S$ par $F$, c'est-à-dire définie par

$$
g(u, u)=\langle F(u), F(u)\rangle
$$

et $B$ l'endomorphisme de $T S$ défini par

$$
g(B(u), u)=\langle A(u), u\rangle .
$$

Rappelons que nous voulons calculer

$$
L(f)=\left.\frac{d}{d t}\right|_{t=0} \operatorname{det}(B) .
$$

Pour cela utilisons le fait que

$$
\left.\frac{d}{d t}\right|_{t=0} g(u, u)=2 f\langle A(u), F(u)\rangle=2 f g(B(u), u) .
$$

En dérivant l'equation $g(B(u), u)=\langle A(u), u\rangle$, nous obtenons

$$
\begin{gathered}
g\left(\left.\frac{d}{d t}\right|_{t=0} B(u), u\right)=\left\langle\left.\frac{d}{d t}\right|_{t=0} A(u), u\right\rangle-f g(B(u), B(u)) \\
=f\langle R(n, u) n, u\rangle-\left\langle\nu_{u} \nu f, u\right\rangle-f g(B(u), B(u))
\end{gathered}
$$

Et donc

$$
\left.\frac{d}{d t}\right|_{t=0} B=f R(n, u) n-\operatorname{Hess}(f)-f B^{2} .
$$

La formule classique

$$
\left.\frac{d}{d t}\right|_{t=0} \log (\operatorname{det}(B))=\operatorname{trace}\left(\left.\frac{d}{d t}\right|_{t=0} B \circ B^{-1}\right),
$$

nous donne la proposition. $\diamond$

Citons un corollaire utile des formules démontrées dans ce paragraphe 
Corollaire 3.1.2 Supposons que $M$ ait une courbure plus petite que-c. Les sphères et les horosphères de $M$ ont alors une courbure plus grande que c. De plus, si $S$ est une surface convexe, pour tout $k<c$, il existe $R$ indépendant de $S$ tel que la surface $S_{R}=\exp (R n(S))$ ait ses courbures principales plus grandes que $k^{1 / 2}$ et en particulier sa courbure plus grande que $k$

\subsection{Démonstration de la proposition 3.0 .3}

Si $\kappa$ est strictement positif, $B$ est défini positif. En particulier, $L$ est elliptique d'indice nul.

Pour conclure, il nous suffit de démontrer que $L$ est injectif. La proposition suivante et une application standard du principe du maximum permettent de conclure :

Proposition 3.2.1 Le terme de degré zéro de la formule de 3.1.1 est strictement positif :

$$
J=\operatorname{trace}\left(W \circ B^{-1}\right)-\operatorname{trace}(B)>0 .
$$

Preuve :utilisons $\left(e_{1}, e_{2}\right)$ une base de vecteurs propres de $B$ associée aux valeurs propres $\lambda_{1}$ et $\lambda_{2}$. Soit $k_{i}$ la courbure du plan engendré par $n$ et $e_{i}$.

Nous obtenons

$$
\begin{aligned}
J & =-\frac{k_{1}}{\lambda_{1}}-\frac{k_{2}}{\lambda_{2}}-\lambda_{1}-\lambda_{2} \\
=1-\lambda_{2}\left(1+\frac{k_{1}}{\kappa}\right)-\lambda_{1}\left(1+\frac{k_{2}}{\kappa}\right)>0 . &
\end{aligned}
$$

$\diamond$

\subsection{Démonstration du lemme 3.0.2}

Pour démontrer la première partie de cette proposition, nous allons procéder par étapes.

Nous résolvons tout d'abord le problème infinitésimal. Une variation infinitésimale de $\Sigma^{t}$, est un champ de vecteur $\zeta$ le long de $\Sigma^{t}$ que nous pouvons écrire sous la forme

$$
\zeta=f n+u
$$

où $u \in T S, f \in C^{\infty}\left(\Sigma^{t}\right)$, et vérifiant la condition au bord

$$
\forall x \in \partial \Sigma, \quad \zeta(x)=\frac{d}{d t} c_{t}(x) .
$$


Ici, nous voyons abusivement $c_{t}$ comme une famille d'immersions de $\partial \Sigma$ dans $M$.

Pour une surface de courbure extrinsèque $\kappa$, la variation infinitésimale de courbure extrinsèque associée à une telle variation $\zeta$ est

$$
L(f)+d \kappa(u),
$$

où $L$ est l'opérateur de 3.0.3.

Pour résoudre infinitésimalement notre problème, nous devons donc montrer qu'il existe une unique fonction $f$ telle que

$$
L(f)=0,
$$

avec la condition au bord

$$
\forall x \in \partial S, f(x)=\left\langle\frac{d}{d t} c_{t}(x), n\right\rangle .
$$

Ceci découle de 3.0.3.

Nous pouvons maintenant utiliser le théorème d'inversion locale pour les opérateurs elliptiques pour résoudre notre problème localement, c'est-à-dire construire au voisinage de 0 une unique famille $t \mapsto \Sigma^{t}$, continue en $t$, de $k$-surfaces vérifiant $\Sigma^{0}=\Sigma$ et $\partial \Sigma^{t}=c_{t}$.

Mettons nous maintenant dans le cadre de la deuxième partie de 3.0.2. Notre lemme 2.2.2 nous permet de montrer que $\Sigma^{t}$ est lentille pour $S^{t}$

Enfin, $S^{t} \subset S^{0}$ pour $t \geq 0$, la proposition 3.2.1, assure que la fonction $f$ décrivant la variation infinitésimale de $\Sigma$ est positive, puisque dans le cas d'une surface lentille nous avons $\left\langle\frac{d}{d t} c^{t}(x), n\right\rangle>0$. Ainsi $\Sigma$ domine $\Sigma^{t}$ pour $t$ positif et suffisamment petit.

\section{Théorème de compacité}

Nous allons dans cette section énoncer et démontrer le théorème de compacité qui nous sera utile par la suite.

Théorème 4.0.1 Soit $M$ une variété d'Hadamard à géométrie bornée et à courbure plus petite que $-c<0$, soit $\left\{\left(S_{n}, x_{n}\right)\right\}_{n \in \mathbb{N}}$ une suite de surfaces convexes immergées à courbure extrinsèque plus grande que c, convergeant vers $\left(S_{\infty}, x_{\infty}\right)$. Nous supposerons de plus cette suite est à géométrie bornée et n'est pas de type horosphérique.

Soit enfin $\{\Sigma\}_{n \in \mathbb{N}}$ une suite de $k_{n}$-surfaces lentilles pour $\left\{S_{n}\right\}_{n \in \mathbb{N}}$, où $k_{n}$ converge vers $k \in] 0, c\left[\right.$, et notons $y_{n} \in \Sigma_{n}$ le pied de $x_{n}$.

Nous avons alors les trois possibilités suivantes 
(i) $S_{\infty}$ n'est pas tubulaire à l'infini et alors, après extraction d'une soussuite $\left\{\left(\Sigma_{n}, y_{n}\right)\right\}_{n \in \mathbb{N}}$ converge vers une $k$-surface lentille non dégénérée pour $S_{\infty}$,

(ii) $S_{\infty}$ n'est pas tubulaire, tout en étant tubulaire à l'infini, alors après extraction d'une sous-suite, $\left\{\left(\Sigma_{n}, y_{n}\right)\right\}_{n \in \mathbb{N}}$ converge vers une $k$-surface lentille éventuellement dégénérée pour $S_{\infty}$,

(iii) $S_{\infty}$ est tubulaire pour une géodésique $\gamma$ et alors $\left\{\left(n\left(\Sigma_{n}\right), n\left(y_{n}\right)\right)\right\}_{n \in \mathbb{N}}$ converge après extraction d'une sous-suite vers le tube de $\gamma$.

Nous allons tout d'abord rappeler les résultats principaux de [3] et leurs conséquences sur ce que nous appelerons le problème de Dirichlet pour les $k$-surfaces. Ensuite, nous appliquerons ces résultats pour démontrer 4.0.1.

\subsection{Rappels sur les problèmes de Monge-Ampère}

\subsubsection{Définitions}

Dans [3], nous avions étudié une classe de problèmes que nous avions appelée de Monge-Ampère et dont les $k$-surfaces forment une classe d'exemple. De plus, nous y avions défini une classe de problème à bord convexe correspondant, dans notre contexte, à la situation suivante.

Un problème de Dirichlet pour les $k$-surfaces est la donnée d'un quadruplet $(M, S, c, x)$, où $c$ est une courbe plongée compléte, tracée sur une surface plongée $S$ localement convexe, incluse dans une variété riemanienne $M$. Le point $x$ sert en particulier à donner un sens à la notion de convergence pour une suite de problèmes de Dirichlet.

Une solution du problème de Dirichlet est une $k$-surface $\Sigma$ complète connexe immergée passant par $x$ telle que $\partial \Sigma \subset c$ et $\Sigma$ est intérieure à $S$ le long de $c$.

Une solution dégénérée est une $k$-surface $\Sigma$ dont le bord $\partial \Sigma$ est complet et inclus dans $c$, telle $\Sigma$ est intérieure à $S$ le long de $c$ et telle qu'enfin $n(\Sigma) \subset U M$ soit complète, sans que $\Sigma$ le soit. Remarquons que dans ce cas, toute surface équidistante de $\Sigma$ est complète.

Pour des raisons techniques, nous sommes obligés d'introduire la définition suivante : si $U$ est un ouvert de $M$ contenant $x$, le problème $(U, S \cap U, c \cap U, x)$ sera appelé problème restreint à $U$ et la restriction à $U$ d'une solution $\Sigma$ définie sur $M$ est la composante connexe de $\Sigma \cap U$ contenant $x$. 


\subsubsection{Remarques}

(i) un tube est une surface rideau au sens de [3] ;

(ii) une $k$-surface lentille $\Sigma$ pour $S$ est un cas particulier de solution du problème de Dirichlet défini par $S$ et $\partial S$;

(iii) Si la courbure moyenne d'une solution est bornée, alors la solution est non dégénérée ;

(iv) Si $S$ est une $k$-surface dégénérée, alors toute surface équidistante est complète.

(v) enfin, il se peut que $S, c$ ou $\partial \Sigma$ soient vide.

\subsubsection{Compacité}

Pour énoncer le résultat principal de [3], il nous faut introduire une notation : pour tout $\varepsilon>0$ et problème de Dirichlet $\Delta, \Delta^{\varepsilon}$ désignera le problème restreint à la boule ouverte de centre $x$ de rayon $\varepsilon$. De même, $\Sigma^{\varepsilon}$ désignera la restriction d'une solution $\Sigma$ à cette boule.

Nous avons alors le

Théorème 4.1.1 Soit $\left\{\Delta_{n}=\left(M_{n}, S_{n}, c_{n}, x_{n}\right)\right\}_{n \in \mathbb{N}}$ une suite de problèmes de Dirichlet convergeant $C^{\infty}$ sur tout compact vers un problème de Dirichlet

$$
\Delta_{\infty}=\left(M_{\infty}, S_{\infty}, c_{\infty}, x_{\infty}\right)
$$

et soit $\left\{\Sigma_{n}\right\}_{n \in \mathbb{N}}$ une suite de $k_{n}$-solutions, éventuellement dégénérée de $\left\{\Delta_{n}\right\}_{n \in \mathbb{N}}$ où $\left\{k_{n}\right\}_{n \in \mathbb{N}}$ converge vers $\left.k \in\right] 0, c[$. Il existe alors $\epsilon$, tel que nous ayions l'alternative suivante, après extraction d'une sous-suite :

(a) soit $\left\{\Sigma_{n}^{\varepsilon}\right\}_{n \in \mathbb{N}}$ converge vers une $k$-solution, éventuellement dégenérée, du problème de Dirichlet $\Delta_{\infty}^{\varepsilon}$;

(b) soit $\left\{n\left(\Sigma_{n}^{\varepsilon}\right)\right\}_{n \in \mathbb{N}}$ converge vers un tube. Dans ce cas, à partir d'un certain rang $\partial \Sigma_{n}^{\varepsilon}=\emptyset$.

Enfin, nous tirons de ce théorème les corollaires suivant, particulier à la courbure négative

Corollaire 4.1.2 Soit $\left\{\Delta_{n}=\left(M_{n}, S_{n}, c_{n}, x_{n}\right)\right\}_{n \in \mathbb{N}}$ une suite de problèmes de Dirichlet convergeant $C^{\infty}$ sur tout compact vers un problème de Dirichlet

$$
\Delta_{\infty}=\left(M_{\infty}, S_{\infty}, c_{\infty}, x_{\infty}\right)
$$


et soit $\left\{\Sigma_{n}\right\}_{n \in \mathbb{N}}$ une suite de $k_{n}$-solutions, éventuellement dégénérée de $\left\{\Delta_{n}\right\}_{n \in \mathbb{N}}$, où $\left\{k_{n}\right\}_{n \in \mathbb{N}}$ converge vers $\left.k \in\right] 0, c[$. Alors la suite de surfaces

$$
W_{n}=\left\{\exp (n(s)), \quad s \in \Sigma_{n}\right\}
$$

pointée en exp $\left(n\left(x_{n}\right)\right)$ converge $C^{\infty}$ après extraction d'une sous suite.

Preuve :ceci provient de ce que la restriction de l'exponentielle à un tube est une immersion à valeur dans $M . \diamond$

Corollaire 4.1.3 Soit $\left\{\left(f_{n}, S\right)\right\}_{n \in \mathbb{N}}$ une suite de surfaces immergées localement convexes convergeant $C^{\infty}$ sur tout compact vers une surface immergée $\left(f_{0}, S\right)$ et soit $\left\{l_{n}\right\}_{n \in \mathbb{N}}$ une suite de fonctions positives définies sur $S$ dont les graphes sont des $k$-surfaces. On suppose que pour tout $y \in S$, la suite $\left\{l_{n}(y)\right\}_{n \in \mathbb{N}}$ est bornée, alors après extraction d'une sous-suite, la suite de fonctions $\left\{l_{n}\right\}_{n \in \mathbb{N}}$ converge $C^{\infty}$ sur tout compact de $S$ vers une fonction dont le graphe est une $k$-surface

Preuve :il suffit pour cela d'appliquer le théorème de compacité 4.1.1 pour la suite de problèmes de Dirichlet (à bord vide) définie dans les bouts des surfaces $f_{n}(S) . \diamond$

\subsection{Une première majoration}

Nous nous donnons donc à partir de maintenant une suite $\{\Sigma\}_{n \in \mathbb{N}}$ de $k_{n^{-}}$ surfaces lentilles pour $\left\{S_{n}\right\}_{n \in \mathbb{N}}$. On suppose de plus que $\left\{S_{n}\right\}_{n \in \mathbb{N}}$ converge vers $S_{\infty}$ qui est à géométrie bornée.

Soit $\{\lambda\}_{n \in \mathbb{N}}$ la suite de fonctions associées définies sur $N_{\Sigma_{n}}$ l'ensemble des vecteurs normaux étendus à $\Sigma_{n}$.

Nous voulons tout d'abord montrer

Proposition 4.2.1 Si la suite $\left\{S_{n}\right\}_{n \in \mathbb{N}}$ n'est pas de type horosphérique, alors la suite de fonctions $\{\lambda\}_{n \in \mathbb{N}}$ est bornée

Preuve :raisonnons par l'aburde et supposons que $\Lambda_{n}$, le maximum de $\lambda_{n}$, tende vers l'infini. Il existe alors deux suites de points $\left\{w_{n} \in S_{n}\right\}_{n \in \mathbb{N}}$ et $\left\{v_{n} \in\right.$ $\left.N_{\Sigma_{n}}\right\}_{n \in \mathbb{N}}$, où $N_{\Sigma_{n}}$ désigne l'ensemble des vecteurs normaux étendus à $\Sigma_{n}$ telles que

(i) $w_{n}=\exp \left(\lambda_{n}\left(v_{n}\right) v_{n}\right)$;

(ii) la suite $\left.\left\{\lambda_{n}\left(v_{n}\right)-\Lambda_{n}\right)\right\}_{n \in \mathbb{N}}$ tende vers zéro.

Notons, pour tout $R, S_{n}^{R}$, la composante connexe de l'intersection de $S_{n}$ avec la boule ouverte de $M$ de centre $w_{n}$ et de rayon $R$. Notons $U_{n}$ le champ de vecteur focal le long de $S_{n}$. 
La suite $\left\{S_{n}\right\}_{n \in \mathbb{N}}$ étant à géométrie bornée, en extrayant au besoin une soussuite, nous pouvons supposer que $\left\{S_{n}^{R}, w_{n}\right\}_{n \in \mathbb{N}}$ converge.

Les fonction inverses $\mu_{n}$ définies sur $S_{n}$ étant 2-lipschtziennes par le lemme 2.2.1, nous pouvons extraire une sous-suite telle que la suite de fonctions $\left\{\mu_{n}-\right.$ $\left.\Lambda_{n}\right\}_{n \in \mathbb{N}}$ converge uniformément vers une fonction 2-lipschtzienne négative $g$. En particulier $\left\{\mu_{n}\right\}_{n \in \mathbb{N}}$ converge uniformément vers l'infini sur $S_{n}^{R}$.

Nous en tirons deux conséquences :

(a) pour tout $L$, il existe $n_{0}$, tel que si $n \geq n_{0}$ alors $d_{i}\left(y_{n}, \partial S_{n}^{R}\right) \geq L$ ( où $d_{i}$ désigne la distance riemanienne dans $S_{n}$ ) puisque la restriction de $\mu_{n}$ au bord de $\Sigma_{n}$ est nulle

(b) à partir d'un certain rang, pour tout $0<t<2 R$ et $z, w \in S_{n}^{R}$, $d\left(\exp \left(-t U_{n}(z),-t U_{n}(w)\right) \leq d(z, w)\right.$.

Rappelons que dans une variété à courbure négative, une hypersurface sans bord localement convexe et complète respectivement à une boule, est plongée et borde un convexe.

En utilisant (a) et ce rappel, nous en déduisons que $\left\{S_{n}, v_{n}\right\}_{n \in \mathbb{N}}$ converge sur tout compact vers une hypersurface plongée globalement convexe $(H, v)$.

Grâce à (b), en extrayant une sous-suite de telle sorte que $\left\{U_{n}\left(v_{n}\right)\right\}_{n \in \mathbb{N}}$ converge vers un vecteur $U$, nous en déduisons que $\left\{U_{n}\right\}_{n \in \mathbb{N}}$ converge sur tout compact vers le champ de gradient de la fonction de Buseman $f$ associée à $\exp (-\infty U)$ qui va se retrouver transverse à $H$.

Par construction $\left.f\right|_{H}-f(v)=g-g(v)$, et nous en déduisons que $\left.f\right|_{H}-f(v) \leq$ $g(v)=0$, et $H$ est de donc de type horosphérique, ce qui est interdit par hypothèse. $\diamond$

\subsection{Surfaces tubulaires et tubes}

Introduisons une définition intermédiaire.

Soit $\left\{w_{n}\right\}_{n \in \mathbb{N}}$ une suite convergente de points de $\left\{S_{n}\right\}_{n \in \mathbb{N}}$. Notons alors $y_{n} \in$ $\Sigma_{n}$ le point pied associé à $w_{n}=\exp \left(\lambda_{n}\left(u_{n}\right) u_{n}\right),\left(c f\right.$ 2.2.2) et $\Sigma_{n}^{\varepsilon}$ la composante connexe de $\Sigma_{n} \cap B\left(y_{n}, \varepsilon\right)$, où $\varepsilon$ est fourni par le théorème 4.1.1de compacité sur les problèmes de Monge-Ampère. Nous dirons que la suite $\left\{w_{n}\right\}_{n \in \mathbb{N}}$ est critique, si après extraction d'une sous suite, $\left.\left\{n\left(\Sigma_{n}^{\varepsilon}\right), u_{n}\right)\right\}_{n \in \mathbb{N}}$ converge vers un tube, c'est-à-dire si nous sommes dans le deuxième cas de l'alternative de 4.1.1, nous dirons de plus dans ce cas, par abus de langage, que la suite critique converge vers $N(\gamma)$. Remarquons que dans ce cas, d'après le théorème cité, à parir d'un certain rang $y_{n}$ est un point intérieur à $S_{n}$ et $u_{n}$ est le vecteur normal à $y_{n}$.

Nous démontrerons deux propositions, nous avons tout d'abord 
Proposition 4.3.1 Si $\left\{S_{n}\right\}_{n \in \mathbb{N}}$ n'est pas de type horosphérique et s'il existe une suite critique, alors la surface $S^{\infty}$ est tubulaire

Preuve :raisonnons par l'absurde et supposons nous donnée une suite critique $\left\{w_{n}\right\}_{n \in \mathbb{N}}$. Soit $y_{n}$ la suite de points pieds associés dans $\{\Sigma\}_{n \in \mathbb{N}}$, et posons $u_{n}=n\left(y_{n}\right) \in n\left(\Sigma_{n}\right)$ Le théorème 4.1.1 assure alors que $\left\{n\left(\Sigma_{n}\right), u_{n}\right\}_{n \in \mathbb{N}}$ converge en tant que variété immergée pointée vers un revêtement $\bar{N}(\gamma)$ d'un tube pointé $N(\gamma)$. Comme $\left\{S_{n}\right\}_{n \in \mathbb{N}}$ elle même converge, la suite de fonctions inverse $\left\{\mu_{n}\right\}_{n \in \mathbb{N}}$ va elle aussi converger sur tout compact vers la fonction fonction $d_{\gamma}$ distance à cette géodésique qui sera bornée par d'après 4.2.1. Par construction, le champ de gradient de $d_{\gamma}$ sera dirigé ver l'intérieur de $S^{\infty}$. Par définition, $S^{\infty}$ est tubulaire. $\diamond$

Réciproquement, nous avons

Proposition 4.3.2 Si $\left\{S_{n}\right\}_{n \in \mathbb{N}}$ est à géométrie bornée et n'est pas de type horosphérique, si $S_{\infty}$ est tubulaire pour $\gamma$ alors toute suite convergente est critique et converge vers $N(\gamma)$

Preuve :soit $\gamma$ la géodésique associée à $S_{\infty}$ et $\mu$ la fonction distance à cette géodésique. Raisonnons par l'absurde et soit $\left\{w_{n}\right\}_{n \in \mathbb{N}}$ une suite convergente de points non critique. Notons comme d'habitude $\left\{y_{n}\right\}_{n \in \mathbb{N}}, y_{n} \in \Sigma_{n}$ la suite de points pieds associées. En utilisant le théorème 4.1.1, nous pouvons donc extraire une sous-suite telle que $\left\{\left(\Sigma_{n}, y_{n}\right)\right\}_{n \in \mathbb{N}}$ converge vers une $k$-surface, $\Sigma_{\infty}$, éventuellement dégénérée.

D'après la proposition 4.2.1 et le fait que $S_{\infty}$ est tubulaire nous en déduisons que $\mu$ est bornée sur $\Sigma_{\infty}$. Soit $\Lambda$ sa borne supérieure.

Construisons maintenant une suite de points $\left\{s_{n}\right\}_{n \in \mathbb{N}}, s_{n} \in \Sigma_{\infty}$ telle que $\mu\left(s_{n}\right)$ tende vers $\Lambda$. La variété $M$ étant à géométrie bornée ainsi que la suite $\left\{S_{n}\right\}_{n \in \mathbb{N}}$, nous pouvons extraire une sous suite telle que

$$
\left\{\Delta_{n}=\left(M, s_{n}, \partial S_{n}, \partial S_{n}\right)\right\}_{n \in \mathbb{N}}
$$

converge vers $\Delta_{0}=\left(M_{0}, s_{0}, \partial S_{0}, S_{O}\right)$.

Nous voulons maintenant appliquer notre théorème de compacité 4.1.1 à la suite $\left\{\left(\Sigma_{\infty}, s_{n}\right)\right\}_{n \in \mathbb{N}}$ solutions des problèmes de Dirichlet $\Delta_{n}$.

Nous avons donc a priori deux possibilités,

(a) soit $\left\{\left(\Sigma_{\infty}, s_{n}\right)\right\}_{n \in \mathbb{N}}$ converge vers une $k$-surface, éventuellement dégénérée,

(b) soit $\left\{\left(n\left(\Sigma_{\infty}\right), n\left(s_{n}\right)\right)\right\}_{n \in \mathbb{N}}$ converge vers un tube $N(\bar{\gamma})$.

Eliminons (b) : puisque $\mu$ est bornée sur $\Sigma_{\infty}$, nécessairement $\bar{\gamma}$ et $\gamma$ sont à distance bornée. Ces deux géodésiques ont alors confondues. Autrement dit, $\Lambda$ est 
nulle ce qui entraîne que la restriction de $\mu$ est nulle sur $\Sigma_{\infty}$. Ceci est impossible pour une $k$-surface.

Ainsi nous ne pouvons être que dans le cadre de (a) et $\left\{\left(\Sigma_{\infty}, s_{n}\right)\right\}_{n \in \mathbb{N}}$ converge vers une $k$-surface, éventuellement dégénérée, $\left(\bar{\Sigma}_{\infty}, s_{\infty}\right)$. La fonction $\mu$ restreinte à $\bar{\Sigma}_{\infty}$ atteint son maximum en $s_{\infty}$ par construction. Ceci signifie que $\bar{\Sigma}_{\infty}$ est intérieurement tangente à la surface convexe $G_{\Lambda}$ à distance constante $\Lambda$ de $\gamma$. Ceci est impossible à cause du principe du maximum géométrique puisque $G_{\Lambda}$ est à courbure extrinsèque strictement plus grande que $-c$.

Nous venons de montrer que toute suite convergente est critique. Par ailleurs, si une suite critique converge vers $N(\bar{\gamma})$, nous avons $\bar{\gamma}=\gamma$ puisque $\mu$ est bornée,. $\diamond$

\subsubsection{Démonstration du théorème 4.0.1}

Soit donc $\left\{S_{n}\right\}_{n \in \mathbb{N}}$ une suite de surfaces à courbure supérieure à $c$, pointée en $\left\{x_{n}\right\}_{n \in \mathbb{N}}$, et convergeant vers $S_{\infty}$ pointée en $x_{\infty}$. Soit enfin $\{\Sigma\}_{n \in \mathbb{N}}$ une suite de $k$ surfaces lentilles pour $\left\{S_{n}\right\}_{n \in \mathbb{N}}$ et $\left\{y_{n}\right\}_{n \in \mathbb{N}}$ la suite des pieds de $\left\{x_{n}\right\}_{n \in \mathbb{N}}$. On suppose que la suite $\left\{S_{n}\right\}_{n \in \mathbb{N}}$ n'est pas de type horosphérique et est à géométrie bornée.

Si $S_{\infty}$ est tubulaire, la proposition 4.3 .2 est exactement (iii) de 4.0.1.

Supposons maintenant que $S_{\infty}$ n'est pas tubulaire. D'après la proposition 4.3.1, aucune suite convergente de points de $\left\{S_{n}\right\}_{n \in \mathbb{N}}$ n'est critique. Le théorème 4.1.1 nous permet donc d'assurer que la suite $\{\Sigma\}_{n \in \mathbb{N}}$ pointée en $\left\{y_{n}\right\}_{n \in \mathbb{N}}$, converge vers une solution éventuellement dégénérée $\Sigma_{\infty}$ du problème de Dirichlet pour $S_{\infty}$. Il nous reste à montrer que $\Sigma_{\infty}$ est lentille pour $S_{\infty}$.

Notons comme précédemment $\left\{\lambda_{n}\right\}_{n \in \mathbb{N}}$ la suite de fonctions associées convergeant vers $\lambda_{\infty}$, pour conclure il suffit de démontrer :

(a) $\lambda_{\infty}$ est strictement positive sur $S_{\infty}$;

(b) si $u$ est le vecteur normal intérieur à $\partial S_{\infty}$ dans $S_{\infty}$ et si $n$ est le champ de vecteur normal extérieur à $\Sigma_{\infty}$ alors $\langle u, n\rangle>0$ le long de $\partial S_{\infty}$.

La preuve de (a) est simple : nous savons déjà que $\lambda_{\infty}$ est positive ou nulle puisque les $\lambda_{n}$ le sont. Si maintenant, $\lambda_{\infty}$ est nulle en un point intérieur à $S_{\infty}$ nous en déduisons que $\Sigma_{\infty}$ est tangente intérieurement à $S_{\infty}$ en ce point ce qui est impossible à cause des conditions de courbure et du principe du maximum géométrique 2.5.1.

Pour (b), remarquons que, par passage à la limite $\langle u, n\rangle \geq 0$ le long de $\partial S_{\infty}$. Si en un point $z,\langle u, n\rangle=0$, alors les deux surfaces sont tangentes en ce point $z$. Elles ne peuvent être tangentes extérieurement : si deux surfaces convexes sont tangentes extérieurement en un point, alors au moins au voisinage de ce point leur intersection est réduite à ce point. Rappelons maintenant que chaque $S_{n}$ est un graphe au dessus 
d'un ensemble fermé du normal étendu à $\Sigma_{n}$. Ceci va être également vrai à la limite; puisqu'enfin en $z$ les surfaces sont tangentes, $\Sigma_{\infty}$ est tangente intérieurement à $S_{\infty}$, ce qui est impossible à cause des hypothèses de courbure et du principe du maximum géométrique.

Enfin, 4.3.1 et 4.3 .2 montre que $S_{\infty}$ est tubulaire à l'infini, si et seulement si il existe une suite $\left\{w_{n}\right\}_{n \in \mathbb{N}}$ telle que $\left\{M, w_{n}\right\}_{n \in \mathbb{N}}$ converge et $\left\{\Sigma_{\infty}, w_{n}\right\}_{n \in \mathbb{N}}$ est critique. En particulier, si $S_{\infty}$ n'est pas tubulaire à l'infini alors la courbure moyenne des $\Sigma_{n}$ est uniformément bornée. $\diamond$

\section{Problème de Plateau pour les disques}

La démonstration de notre lemme de Morse va se faire par étapes et la première d'entre elles est la résolution du problème de Plateau pour les disques.

Nous allons en fait démontrer un résultat plus précis qui est le but de cette section :

Proposition 5.0.3 Soit $S$ un disque compact immergé à courbure $>c$, dans une variété d'Hadamard à courbure plus petite que $-c$. Alors, pour tout $k \in] 0, c[$, il existe une unique $k$-surface lentille $\Sigma$ pour $S$.

De plus, si $c>k_{1} \geq k>0$, si $\Sigma_{1}$ est la $k_{1}$-surface lentille pour un disque $S_{1} \subset S$, alors $\Sigma$ domine $\Sigma_{1}$ au sens de 5.3.1.

Nous allons utiliser une méthode de déformation.

\subsection{Déformation contractante}

Soit $k \in] 0, c\left[\right.$. Notons $f_{1}$ l'immersion du disque $S$ dans $M$. Identifions d'une manière ou d'une autre ce disque à la boule unité de $\mathbb{R}^{2}$. Soit alors $\left.\left.f_{t}, t \in\right] 0,1\right]$, la famille d'immersions de $S$ dans $M$ définie par $f_{t}(x)=f_{1}(t x)$.

Le but de ce paragraphe est de démontrer la

Proposition 5.1.1 Soit $\Sigma$ une $k$-surface lentille pour $f_{1}$, et $k(t)$ une fonction croissante à valeurs dans $] 0, c[$ telle que $k(0)=k$. Il existe alors une unique famille continue de $k(t)$-surfaces lentilles $\Sigma_{t}$ pour $f_{t}(S)$, définie pour tout $\left.\left.t \in\right] 0,1\right]$ et vérifiant $\Sigma_{1}=\Sigma$. Enfin, quand $t$ tend vers $0, \Sigma_{t}$ tend vers $f_{1}(0)$ au sens de la topologie de Haussdorff pour les compacts

Preuve :nous utilisons la proposition 3.0 .2 pour construire au voisinage de 1 une famille de déformations de $\Sigma_{1}$. D'après la deuxième partie de cette assertion, nous obtenons de plus que si $t>s$ alors $\Sigma_{s}$ domine $\Sigma_{t}$. 
Supposons nous donnée une déformation de $\Sigma_{1}$ pour $t$ dans un intervalle $\left.] a, 1\right]$. Pour conclure la démonstration de la première partie dela proposition 5.1.1, il nous reste à montrer que pour toute suite $\left\{a_{n}\right\}_{n \in \mathbb{N}}$ tendant vers $a$ par valeurs supérieures, alors la suite $\left\{\Sigma_{a_{n}}\right\}_{n \in \mathbb{N}}$ converge vers une surface lentille pour $f\left(S_{a}\right)$.

Le corollaire de notre théorème de compacité 4.0.1 nous permet d'affirmer qu'après extraction éventuelle d'une sous suite, $\left\{\Sigma_{a_{n}}\right\}_{n \in \mathbb{N}}$ converge vers une $k$ surface.

Enfin si $\left\{a_{n}\right\}_{n \in \mathbb{N}}$ et $\left\{\bar{a}_{n}\right\}_{n \in \mathbb{N}}$ sont deux suites convergeant vers $a$ par valeurs supérieures, telles que les suites $\left\{\Sigma_{a_{n}}\right\}_{n \in \mathbb{N}}$ et $\left\{\Sigma_{\bar{a}_{n}}\right\}_{n \in \mathbb{N}}$ convergent respectivement vers $\Sigma_{a}$ et $\Sigma_{\bar{a}}$, nous obtenons que $\Sigma_{a}$ domine $\Sigma_{\bar{a}}$ et réciproquement. Ainsi $\Sigma_{a}=\Sigma_{\bar{a}}$, ce qui achève de démontrer que si $t$ tend vers $a$ par valeurs inférieures, $\Sigma_{t}$ converge.

Ce dernier point termine la démonstration de la première partie de notre proposition.

La deuxième partie de la poposition découle immédiatement de la proposition 2.5.2 qui affirme : si $S$ est une surface compacte dont le bord $\partial S$ est incluse dans une boule $B$, alors toute $k$-surface $\Sigma$, pour $k \leq c$, solution du problème de Dirichlet pour $S$ est incluse dans la boule $B . \diamond$

\subsection{Unicité}

Nous pouvons maintenant montrer

Proposition 5.2.1 Soit $S$ un disque compact immergé à courbure $>c, k_{1}$ et $k$ tels que $0<k \leq k_{1}<c$. Si $\Sigma$ est une $k$-surface lentille pour $S$, et $\Sigma_{1}$ une $k_{1}$-surface lentille pour $S_{1} \subset S$, alors $\Sigma$ domine $\Sigma_{1}$.

En particulier, il existe au plus une $k$-surface lentille pour $S$.

Preuve :considérons comme dans le paragraphe précédent, $f_{1}$ l'immersion de notre disque $S_{1}$ et $f_{t}$ la famille contractante d'immersions associée, soit de plus $k(t)$ une fonction strictement décroissante à valeurs dasn ]0, $c$, telle que $k_{1}=k(1)$.

Soit $\Sigma_{1}$ comme dans l'énoncé ; la proposition 5.1.1 nous permet de lui associer une famille de $k_{1}$-surfaces lentilles $\Sigma_{t}$ pour $f_{t}$. Pour montrer que $\Sigma$ domine $\Sigma_{1}$, il suffit de montrer que $\Sigma$ domine $\Sigma_{t}$ pour tout $t$.

En vertu de notre lemme de domination 2.6.1 et du principe du maximum géométrique 2.5.1, il suffit en fait de montrer qu'il existe $t$, tel que $\Sigma$ domine strictement $\Sigma_{t}$. Mais ceci découle de la dernière partie de la proposition 5.1.1: clairement, puisque $\Sigma_{t}$ converge vers un point dans la topologie de Haussdorf quand $t$ tend vers $0, \Sigma$ domine $\Sigma_{t}$, pour $t$ suffisamment petit. $\diamond$ 


\subsection{Existence}

Pour conclure la démonstration de 5.0.3, il nous faut montrer l'existence d'une surface lentille pour notre disque immergé $S$, nous allons pour cela construire une nouvelle famille de déformations de ce disque.

Ce disque étant compact, il est inclus dans une boule $B$. Nous pouvons alors pertuber la métrique de $M$ dans un petit ouvert $U$ n'intersectant pas cette boule $B$ de façon à ce que la nouvelle métrique soit toujours à courbure plus petite que $-c$, et que de plus elle soit à courbure constante dans une boule $B_{0} \subset U$.

Il suffit de montrer de montrer l'existence d'une surface lentille pour la métrique perturbée puisque d'après 2.5.2, cette surface lentille sera incluse dans $B$ et sera donc lentille pour la métrique originelle.

Il est maintenant facile mais technique de montrer le lemme

Lemme 5.3.1 Soit $S$ un disque immergé à courbure extrinsèque plus grande que $c_{1}$, $i l$ existe alors une déformation continue $S_{t}, S_{0}=S$ de ce disque $t \in[0,1]$, telle que

(i) pour tout $t$, la courbure extrinsèque de $f_{t}$ est plus grande que $c_{1}$;

(ii) $S_{1} \subset B_{0}$ est à courbure constante, et son bord est inclus dans un plan totalement géodésique de $B_{0}$.

Preuve :nous allons donner une esquisse de la construction de cette déformation. Nous utilisons tout d'abord la déformation contractante pour déformer notre disque en en tout petit disque inclus dans le bord d'une surface convexe. Ce petit disque va alors être un graphe au dessus d'un disque topologique sur une sphère de petit rayon. Ensuite nous déplaçons cette sphère de telle sorte quelle soit incluse dans $B_{0}$ et le tour est joué.»

Utilisons cette déformation pour construire une $k$-surface lentille pour $S$.

En utilisant les équidistantes aux plans totalement géodésiques dans l'espace hyperbolique, nous construisons une $k$-surface lentille pour $S_{1}$.

Notre lemme 3.0.2 nous permet de construire une famille continue de $\mathrm{k}$ surfaces lentilles $\Sigma_{t}$ pour $S_{t}$ sur un intervalle maximal ]a,1]. Le théorème de compacité 4.0.1 et l'unicité 5.2.1, entraînent que $\Sigma_{t}$ converge lorsque $t$ tend vers $a$; ainsi $a=0$ et nous avons terminé notre démonstration. 


\section{Démonstration du lemme de Morse pour les surfaces convexes}

Dans toute cette section, $S$ désignera une surface localement convexe, éventuellement à bord, à courbure plus grande que $c$, à géométrie bornéee et qui n'est ni horosphérique à l'infini, ni tubulaire, ni compacte sans bord.

Le but de cette section est le lemme suivant, que nous appelons lemme de Morse pour les surfaces convexes.

Lemme 6.0.2 Soit $M$ une variété d'Hadamard à géométrie bornée et à courbure strictement plus petite que $-c<0$. Soit $S$ une surface localement convexe, éventuellement à bord, à courbure plus grande que c, à géométrie bornéee et qui n'est ni horosphérique à l'infini, ni tubulaire, ni compacte sans bord. Alors, pour tout $k \in] 0, c[$, il existe une unique $k$-surface lentille, éventuellement dégénérée, pour $S$.

De plus, si $S$ n'est pas tubulaire à l'infini, cette $k$-surface est non dégénérée.

Il suffit de démontrer ce résultat quand $S$ est un disque topologique : dans le cas général, il suffit en effet de passer au revêtement universel et d'utiliser l'unicité pour conclure.

Nous supposerons donc dans toute la suite que $S$ est un disque. Notre lemme de Morse est une conséquence immédiate des deux propositions suivantes 6.1.1 et 6.2.1, où nous démontrons successivement l'existence et l'unicité d'une $k$-surface lentille pour $S$.

\subsection{Existence}

Nous allons montrer la proposition :

Proposition 6.1.1 Il existe une $k$-surface lentille $\Sigma$ pour $S$, telle que pour toute $k$-surface lentille $\bar{\Sigma}$ pour $S, \bar{\Sigma}$ domine $\Sigma$. De plus si $S$ n'est pas tubulaire à l'infini, $\Sigma$ est non dégénérée.

Preuve :considérons $S_{t}$ une famille continue de disques inclus dans $S$ définie pour $t \in] 0,1]$ et vérifiant

(i) $S_{1}=S$;

(ii) $S_{t}$ est compact à bord pout $t \neq 1$;

(iii) $S_{t} \subset S_{s}$ si $t \leq s$;

(iv) $S_{t}$ converge vers un point $x_{0}$ quand $t$ tend vers 0 . 
La proposition 5.0.3 nous permet donc de construire une famille $\Sigma_{t}$ de $k$-surfaces lentilles pour $S_{t}$. par ailleurs, notre théorème de compacité 4.1.1, nous permet d'extraire une suite $\left\{t_{n}\right\}_{n \in \mathbb{N}}$ tendant vers 1 , telle que $\left\{\Sigma_{t_{n}}, y_{n}\right\}_{n \in \mathbb{N}}$ converge vers une $k$-surface lentille pointée $\Sigma$ quand $n$ tend vers l'infini. Ici, $\left\{y_{n}\right\}_{n \in \mathbb{N}}$ désigne bien sûr la suite de points pieds de $x_{0}$ dans $\Sigma_{t_{n}}$.

Si maintenant $\bar{S}$ est une autre surface lentille, il est clair que $\bar{\Sigma}$ domine $\Sigma_{t}$ pour $t$ petit. Ensuite notre lemme 2.6.1 et le principe du maximum 2.5.1 assure que $\bar{\Sigma}$ domine strictement $\Sigma_{t}$ domine $t$ pour tout $t$. Ainsi $\bar{\Sigma}$ domine-t-elle également $\Sigma$. $\diamond$

\subsection{Unicité}

L'unicité d'une $k$-surface lentille découle immédiatement de la proposition précédente 6.1.1 et de

Proposition 6.2.1 Si $\Sigma_{1}$ et $\Sigma_{2}$ sont deux $k$-surfaces lentilles pour $S$ telles que $\Sigma_{1}$ domine $\Sigma_{2}$ alors $\Sigma_{1}=\Sigma_{2}$

Preuve :soit $N_{\Sigma_{1}}$ le fibré normal étendu. Soit $F$ l'immersion naturelle de $M_{1}=$ $N_{\Sigma_{1}} \times \mathbb{R}^{+}$dans $M$ qui à $(n, t)$ associe $\exp (t n)$. Notons $\mu$ la fonction de $M_{1}$ qui a $(n, t)$ associe $t, G_{\lambda}$ la surface localement convexe de niveau $\lambda$ pour la fonction $\mu$, et enfin $\pi$ la projection de $M_{1}$ dans $\Sigma_{1}$.

Par définition, puisque $\Sigma_{1}$ domine $\Sigma_{2}$, nous pouvons trouver une immersion $i$ de $\Sigma_{2}$ dans $M_{1}$, telle que $F \circ i$ est l'immersion inititale de $\Sigma_{2}$.

Confondons $\Sigma_{2}$ et son image dans $M_{1}$. Notre but est de montrer que $\mu$ restreinte à $\Sigma_{2}$ est nulle.

Supposons donc le contraire et soit $\left\{w_{n}\right\}_{n \in \mathbb{N}}$ une suite de points de $S_{2}$, telle que $\left\{\mu\left(w_{n}\right)\right\}_{n \in \mathbb{N}}$ converge vers la borne supérieure $\Lambda \neq 0$ de $\mu$ sur $\Sigma_{2}$.

Extrayons de $\left\{w_{n}\right\}_{n \in \mathbb{N}}$ une sous-suite telle que $\left\{M, w_{n}\right\}_{n \in \mathbb{N}}$ converge vers une variété $\left(M_{0}, w_{\infty}\right)$, et utilisons le théorème de compacité 4.1.1 pour extraire extraire de la suite $\left\{w_{n}\right\}_{n \in \mathbb{N}}$ une sous-suite telle que les deux suites $\left.\left\{\Sigma_{1}, z_{n}\right)\right\}_{n \in \mathbb{N}}$ et $\left\{\Sigma_{2}, w_{n}\right\}_{n \in \mathbb{N}}$ convergent. Ici $z_{n}=\pi \circ i\left(w_{n}\right)$ où $\pi$ est la projection naturelle de $M_{1}$ dans $\Sigma_{1}$.

En ce qui concerne $\left\{\Sigma_{1}, z_{n}\right\}_{n \in \mathbb{N}}$, nous avons comme toujours deux possibilités :

- soit $\left\{\Sigma_{1}, z_{n}\right\}_{n \in \mathbb{N}}$ converge vers une $k$-surface,

- soit $\left.\left\{n\left(\Sigma_{1}\right), n\left(z_{n}\right)\right)\right\}_{n \in \mathbb{N}}$ converge vers un tube.

Néanmoins, dans ces deux cas, la suite de surfaces convexes pointées

$$
\left\{G_{\Lambda}, \exp \left(\Lambda n\left(z_{n}\right)\right)\right\}_{n \in \mathbb{N}}
$$


converge vers une surface strictement convexe lisse à courbure strictement plus grande que $k$.

Examinons maintenant $\left\{\Sigma_{2}, w_{n}\right\}_{n \in \mathbb{N}}$, nous avons aussi deux cas.

(a) Soit $\left\{\Sigma_{2}, w_{n}\right\}_{n \in \mathbb{N}}$ converge vers une $k$-surface; le principe du maximum géométrique 2.5.1 exclut cette possibilité puisque cette $k$-surface serait alors tangente intérieurement d'après notre construction à $G_{\Lambda}$.

(b) Soit $\left\{n\left(\Sigma_{2}\right), n\left(w_{n}\right)\right\}_{n \in \mathbb{N}}$ converge vers un tube $N(\gamma)$, ceci est à nouveau exclus puisque alors la géodésique $\gamma$ serait tangente intérieurement à $G_{\Lambda}$ qui est strictement convexe.

Ainsi $\Lambda=0$, et nous avons terminé notre démonstration. $\diamond$

\section{Problèmes asymptotiques}

Rappelons le problème de Plateau asymptotique : nous nous donnons une immersion $i$ d'une surface $S$ dans $\partial_{\infty} M$ le bord à l'infini de $M$, et nous cherchons une immersion $f$ de $S$ dans $M$, telle que

(i) $f(S)$ soit une $k$-surface éventuellement dégénérée

(ii) si $N$ est l'application de Gauss-Minkowski de $f(S)$ dans $\partial_{\infty} M$ qui a un point associe le point à l'infini de la normale extérieure asymptote, alors

$$
i=N \circ f
$$

Le couple $(i, S)$ sera la donnée de ce problème de Plateau asymptotique et $(f, S)$, ou plus simplement $f$, sera une solution de ce problème.

Nous allons résoudre ce problème tout d'abord dans le cas où $S$ est le disque ouvert et où $f$ s'étend en un plongement du disque fermé. Nous utiliserons ce résultat pour montrer l'unicité de la solution du problème de Plateau, puis l'existence dans les cas qui nous intéressent.

\subsection{Disques plongés}

Nous voulons montrer le

Lemme 7.1.1 Soit $f$ un plongement du disque fermé $\bar{D}$ dans le bord à l'infini $\partial_{\infty} M$, alors il existe une unique $k$-surface plongée solution du problème de Plateau à l'infini défini par $f$ restreinte au disque ouvert $D$

Preuve :il suit des constructions de [1] , le fait, désormais classique, qu'il existe une surface convexe $S$, plongée homéomorphe au disque, bordant un convexe $C$ telle 
que l'application de Gauss-Minkowski soit un homéomorphisme de $S$ sur $f(D)$; on considère pour cela $S_{0}$ le bord de l'enveloppe convexe de $\partial_{\infty} M \backslash f(D)$. En remplaçant au besoin cette surface par la surface $S_{\varepsilon_{0}}$ à distance constante $\varepsilon_{0}$ où $\varepsilon_{0}$ ne dépend que de $k$ et pas de $S_{0}$, on peut de plus supposer qu'elle a une courbure strictement plus grande que $k$ ( $c f$. corollaire 3.1.2).

La $k$-surface $\Sigma_{k}$ lentille pour $S_{\varepsilon}$ produite par le lemme de Morse 6.0 .2 est alors une solution du problème asymptotique. Il s'agit maintenant de montrer l'unicité de cette solution que nous appelerons pour le moment canonique. Soit donc $\bar{\Sigma}_{k}$ une autre solution, nous voulons montrer que $\bar{\Sigma}_{k}=\Sigma_{k}$.

Pour cela, nous allons raisonner en deux temps.

(i) Les trois surfaces $\bar{\Sigma}_{k}, \Sigma_{k}$ et $S_{0}$ bordent des convexes que nous noterons $\bar{O}_{k}, O_{k}$ et $U_{0}$ respectivement. Nous voulons montrer que $\bar{\Sigma}_{k}$ se trouve à l'extérieur de $\Sigma_{k}$, c'est-à-dire que $O_{k} \subset \bar{O}_{k}$.

Bien sûr, nous avons

$$
U_{0} \subset \bar{O}_{k}, \quad U_{0} \subset O_{k}
$$

Par construction la surface $\Sigma_{k}$ est coincée entre $S_{\varepsilon}$ (c'est une surface lentille) et $S_{0}$ (elle borde un convexe). En particulier lorsque $k$ tend vers $0, \Sigma_{k}$ tend vers $S_{0}$. Notons $F$ la surface convexe à distance $\varepsilon_{0}$ de $\bar{O}_{k}$; comme $U_{0} \subset \bar{O}_{k}, F$ se trouve à l'extérieur de $O_{k}$.

Soit alors $t \mapsto k_{t}$ une fonction continue strictement décroissante de [0,1] dans $\left[k, k_{0}\right]$ où $k_{0}$ est choisi de telle sorte que la courbure de $F$ soit strictement plus grande que $k_{0}$. Par ailleurs soit $\left.\left.B_{t}, t \in\right] 0,1\right]$ une suite exhaustive continue de compacts à bord lisse de $F, k_{t}$ et $F_{t}$ la suite de $k(t)$-surfaces solutions du problème de Plateau pour $B_{t}$. Par le principe de maximum géométrique et un argument de déformation, $F_{t}$ se trouve toujours à l'extérieur de $O_{k}$. Enfin, lorsque $t$ tend vers 1 , nous avons vu que $F_{t}$ converge vers la solution du problème de Plateau pour $F$, c'est-à-dire $\bar{\Sigma}_{k}$.

Ainsi $\bar{\Sigma}_{k}$ se trouve à l'extérieur de $\Sigma_{k}$.

(ii) Par ailleurs, soit $D^{r}$ le disque de rayon $r$ inclus dans $D$, et $k(r)$ une fonction de $r$ strictement décroissante. notons alors $S_{\varepsilon}^{r}$ à distance constante $\varepsilon$ du bord de l'enveloppe convexe de $\partial_{\infty} M \backslash f\left(D^{r}\right)$ la surface et $\Sigma_{k(r)}^{r}$ la $k(r)$-solution canonique du problème de Plateau asymptotique pour $D^{r}$. Les familles $\left\{S_{\varepsilon}^{r}\right\}_{r \in[0,1]}$ et $\left\{S_{0}^{r}\right\}_{r \in[0,1]}$ dépendent continuement de $r$, ainsi que les surfaces $\left\{\Sigma_{k(r)}^{r}\right\}_{r \in[0,1]}$. Notre deuxième remarque est la suivante : pour $r$ strictement plus petit que $1 \bar{\Sigma}_{k}$ est nécessairement à l'intérieur de $\Sigma_{k(r)}^{r}$. En effet, dans le cas contraire, nous pourrions trouver une surface compacte $U, U \subset \bar{\Sigma}_{k}$ dont le bord serait dans $\Sigma_{k(r)}^{r}$ (les deux surfaces s'intersectant transversalement par le principe de maximum géométrique 
2.5.1) et en particulier borderait $V \subset \Sigma_{k}^{r}$. La surface $V$ serait alors lentille pour $U$. Ceci est impossible : en effet la $k$-surface $W$ lentille pour $V$ serait également lentille pour $U$, et nous aurions ainsi deux $k$-surfaces lentilles pour $U$, à savoir $U$ et $W$.

En faisant tendre $r$ vers 1 , on obtient bien que $\bar{\Sigma}_{k}=\Sigma_{k}$. $\diamond$

Nous allons par la suite avoir besoin d'un lemme plus explicite, utilisant que $M$ est à géométrie bornée. Ce lemme est l'analogue de la $\delta$-hyperbolicité pour les géodésiques, ou du caractère fin des triangles en courbure strictement négative. Pour cela, notons $U_{\alpha}^{(z, u)} \subset M$ le cône d'angle $\alpha$ autour du vecteur $u$ en $z$.

Remarquons de plus que par compacité et géométrie bornée, il existe une constante $0<\alpha_{0}<\pi / 2$ telle que $\alpha \leq \alpha_{0}$ entraîne que, pour tout $z$, u, le point $z$ appartient à l'enveloppe convexe de $\partial_{\infty} M \backslash \partial_{\infty} U_{\alpha}^{(z, u)}$.

Lemme 7.1.2 Soit $0 \leq \beta<\alpha<\alpha_{0}$, il existe une constante $\delta(\alpha, \beta)$ ne dépendant que de $\alpha$ et $\beta$ telle que si $z$ est un point quelconque de $M$, et $S$ la solution du problème de plateau asymptotique pour $\partial_{\infty} U_{\alpha}^{(z, u)}$, alors elle est incluse dans $U_{\alpha}^{(z, u)}$ et toute géodésique joignant $z$ à un point de $\partial_{\infty} U_{\beta}^{(y, u)}$ intersecte $S$ à une distance plus petite que $\delta(\alpha, \beta)$ de $y$

Preuve :ce lemme découle immédiatement du résultat précédent et d'un argument de compacité $\diamond$

\section{$7.2 \quad$ Unicité}

Nous voulons montrer

Théorème 7.2.1 Il existe au plus une solution du problème de Plateau asymptotique. De plus si $(f, S)$ est une $k$-surface (pas nécessairement complète) et si $\Sigma \subset S$, alors il existe une solution du problème de Plateau asymptotique pour $(N, \Sigma)$, où $N$ est l'application de Gauss Minkowski, et cette solution est un graphe au dessus de $(f, \Sigma)$

Ce théorème découle immédiatement des trois propositions suivantes :

Proposition 7.2.2 Supposons que $S$ est un disque, et soient $(f, S)$ et $(g, S)$ deux solutions du problème de plateau asymptotique, il existe alors une troisième solution du problème de Plateau $(h, S)$ qui est un graphe à la fois au dessus de $f(S)$ et $g(S)$.

Proposition 7.2.3 Soit $\Sigma$ une $k$-surface éventuellement dégénérée et soit $\Sigma_{1}$ une $k$-surface qui est un graphe au dessus de $\Sigma$, alors $\Sigma=\Sigma_{1}$ 
L'unicité découle immédiatement de ces deux premières propositions ( en passant au besoin au revêtement universel ), et la dernière partie du théorème du résultat suivant, qui va être l'outil fondamental dans la démonstration des deux premières propositions.

Proposition 7.2.4 Soit $(f, S)$ une $k$-surface éventuellement dégénérée et soit $\Sigma$ un ouvert de $S$. Il existe alors une solution du problème de Plateau asymptotique pour $(N, \Sigma)$, où $N$ est l'application de Gauss Minkowski, telle que cette solution est un graphe au dessus de $(f, \Sigma)$.

\subsubsection{Démonstration de la proposition 7.2.4}

Preuve :examinons tout d'abord le cas où $\Sigma$ est un ouvert à bord lisse et relativement compact de $S$.

Posons $\Sigma_{0}=f(\Sigma)$, et considérons $\Sigma_{r}^{0}$ la surface "à distance $r$ " de $\Sigma_{0}$, c'est à dire, en notant $n$ le champ de vecteur normal extérieur à $f(S)$,

$$
\Sigma_{r}^{0}=\left\{\exp (r n(s)), s \in \Sigma_{0}\right\}
$$

Soit ensuite $\Sigma_{r}$ la solution du problème de Plateau pour $D_{r}$. La famille $\left\{\Sigma_{r}\right\}$ forme alors une famille continue, notre but est bien sûr de montrer que $\left\{\Sigma_{r}\right\}$ converge, quand $r$ tend vers $+\infty$ vers une solution du problème de Plateau asymptotique que nous recherchons.

(i) Montrons tout d'abord que pour tout $r, \Sigma_{r}$ est un graphe au dessus de $\Sigma_{0}$.

Remarquons tout d'abord que ceci est vrai pour $r$ petit. En effet, si $g$ est la fonction correspondant à la variation infinitésimale de $\Sigma_{r}$ en $r=0$, cette fonction satisfait $L(g)=0$ et $\left.g\right|_{\partial \Sigma_{0}}>0$, où $L$ est l'opérateur elliptique défini en 3.0.2. Il suit de 3.2.1 et du principe de maximum que $g>0$.

Pour montrer que pour tout $r, \Sigma_{r}$ est un graphe au dessus de $\Sigma_{0}$, considérons l'ensemble $O$ des $r$ tels que $\Sigma_{r}$ soit un graphe. L'ensemble $O$ est évidemment un ouvert de $\mathbb{R}^{+}$. Soit alors $\left[0, r_{0}\right.$ [ la composante connexe de 0 . Raisonnons par l'absurde et supposons que $r_{0}$ est finie.

Pour $r$ plus petit que $r_{0}$, la surface $\Sigma_{r}$ sépare le bout $B$ de $\Sigma_{0}$ en deux composantes connexes l'une bornée et contenant $\Sigma_{0}$, et l'autre non bornée.

Par continuité, la surface $\Sigma_{r_{0}}$ étant limite de graphes, la normale extérieure à $\Sigma_{r_{0}}$ va toujours pointer vers une composante connexe de $B \backslash \Sigma_{r_{0}}$ ne contenant pas $\Sigma_{0}$. Mais par ailleurs, cette surface n'est pas un graphe et il existe donc un point $x$ de $\Sigma_{0}$ en tel que la normale issue de $x$ est tangent intérieurement à $\Sigma_{r_{0}}$ en un point $y$. Par convexité locale de $\Sigma_{r_{0}}$, la normale extérieur en $y$ serait alors dirigée vers une composante connexe de $B \backslash \Sigma_{r_{0}}$ contenant $x$ d'où la contradiction. 
(ii) Associons donc à $\Sigma_{r}$, la fonction $f_{r}$ dont elle est le graphe. Montrons maintenant que pour tout $y, f_{r}(y)$ est bornée.

Soit $u$ le vecteur normal en $y$, il existe alors $\alpha>0$ petit tel que $U_{\alpha}^{(y, u)}$ soit plongé dans le bout de $\Sigma$. Si $D$ est la solution du problème de Plateau asymptotique pour $\partial_{\infty} U_{\alpha}^{y, u}$, elle va pouvoir être plongée dans le bout de $\Sigma$, nous savons alors que la géodésique issue de $y$ dans la direction de $u$ intersecte $D$ au temps $K$. Par le principe du maximum géométrique la surface $D$ va alors servir de barrière à la famille $\left\{\Sigma_{r}\right\}$, et nous en déduisons que $\forall r, f_{r}(y) \leq K$.

(iv) Nous pouvons maintenant montrer maintenant montrer que la suite $\left\{\Sigma_{r}\right\}$ converge après extraction éventuelle d'une sous-suite vers une surface $\Sigma_{\infty}$ qui est le graphe d'une fonction $\lambda$ définie sur $\Sigma_{0}$ et propre (i.e. tendant vers l'infini lorsque l'on tend vers le bord de $\Sigma_{0}$.

Considérons la suite de fonctions $\left\{f_{r}\right\}$ définies au paragraphe précédent. Pour chaque $y$ la suite $\left\{f_{r(y)}\right\}$ est croissante et bornée. Nous en déduisons immédiatement que cette suite de fonctions converge vers une fonction $\lambda$ propre et de graphe convexe. Pour cela, il faut bien sûr utiliser la classique compacité des convexes en courbure négative. Il nous reste à montrer que le graphe de la limite est une $k$-surface. Ceci découle immédiatement du théorème 4.1.1 appliqué au problème de Dirichlet à bord vide défini sur le bout de $\Sigma_{0}$ et de la remarque suivante : la suite de surfaces $\left\{\Sigma_{r}\right\}$ est toujours transverse au champ de vecteur normal à $\Sigma_{0}$ (ce sont des graphes), et en particulier on ne peut pas voir apparaître de tubes à la limite.

(v) Montrons enfin que la surface limite $\Sigma_{\infty}$ est une solution du problème de Plateau asymptotique pour $\left(N, \Sigma_{0}\right)$. Notons $B$ le bout de $S$. La surface $\Sigma_{\infty}$ va séparer $B$ en deux composantes connexes. Soit $C$ la composante connexe de l'extérieur de $\Sigma_{\infty}$. Par construction $\partial_{\infty} C=N\left(\Sigma_{0}\right)$, en particulier, nous en déduisons que l'application de Gauss-Minkowski de $\Sigma_{\infty}$ est injective et à valeurs dans $\Sigma_{0}$ vu comme sous-ensemble du bord à l'infini de $B$ grâce à l'application de GaussMinkowski de $S$. Il nous reste à montrer que cette application est surjective. Pour cela, il suffit de remarquer que si $v \in N\left(\Sigma_{0}\right)$ et si $h$ est la fonction horosphérique associée à $v$, alors $h$ est propre sur $\Sigma_{\infty}$, elle admet donc un mimimum c'est à dire un point dont la normale pointe vers $v$.

(vi) Il nous reste à examiner le cas où $\Sigma_{0}$ n'est pas à bord lisse et relativement compact. On construit alors une suite $\left\{\Sigma_{r}^{0}\right\}$ d'ouverts à bord lisse et relativement compacts, emboités et dont la réunion est $\Sigma_{0}$. D'après ce que nous venons de voir, nous pouvons alors construire une suite $\left\{\Sigma_{r}\right\}$ de $k$-surfaces dans $B$, le bout de $\Sigma_{0}$, dont les bouts sont emboîtés, et fournissant des solutions des problèmes de Plateau asymptotiques associés à $\left\{\Sigma_{r}^{0}\right\}$. Une adaptation sans douleur de la démonstration 
précédente montre alors que la suite $\left\{\Sigma_{r}\right\}$ va converger vers une $k$-surface solution de notre problème. $\diamond$

\subsubsection{Démonstration de la proposition 7.2.3}

Preuve :Soit $\Sigma$ une $k$-surface éventuellement dégénérée et soit $\Sigma_{1}$ une $k$-surface qui est un graphe au dessus de $\Sigma$. Notons $f$, la fonction associée. Pour montrer que $\Sigma=\Sigma_{1}$, il nous suffit de montrer que $f$ est bornée par une constante $a$. En effet, $\Sigma$ et $\Sigma_{1}$ seront alors toutes les deux lentilles pour la surface $S_{a}$ à distance $a$ de $\Sigma$, et nous aurons l'unicité grâce à la proposition 6.2.1.

Montrons donc que $f$ est bornée. Considérons comme d'habitude le bout $B$ de $\Sigma$, et commençons par remarquer que dans le cas où $\Sigma$ est complète, alors pour tout $y \in \Sigma$ de vecteur normal extérieur $n$ et tout vecteur $u$ tel que $\langle u, n\rangle>0$, la géodésique issu de $u$ reste tracée dans $B$. Cela reste vrai dans le cas d'une $k$-surface dégénérée : en effet une telle géodésique heurte transversalement en un temps fini une surface équidistante, et une telle surface est toujours complète.

En particulier, le cône $U_{\alpha_{0}}^{y, n}$ est inclus dans $B$, où $\alpha_{0}$ est la constante de 7.1 .2 et $n$ désigne vecteur normal à $y$. Soit $D_{y}$ l'unique solution du problème de Plateau asymptotique pour $\partial_{\infty} U_{\alpha_{0}}^{(y, n)}\left(c f\right.$ 7.1.1), ce disque $D_{y}$ est inclus dans $U_{\alpha_{0}}^{y, n}$, d'après 7.1 .2 et en particulier dans $B$; par 7.2.4, c'est donc le graphe d'une fonction $f_{y}$ au dessus d'un ouvert $U_{y}$ relativement compact de $\Sigma$.

Montrons que $f_{y} \geq f$. Dans le cas contraire, soit $V_{y}=\left\{z / f_{y} \geq f\right.$. D'après la propreté de $f_{y}$ sur $U_{y}, V_{y}$ est compact. De plus les deux surfaces s'intersectant transversalement (à cause du principe du maximum géométrique) $V_{y}$ est à bord lisse. Il est alors facile de voir que le graphe de $f_{y}$ restreint à $V_{y}$ est lentille pour celui de $f$, ce qui est impossible d'après 6.2.1.

Ainsi $f(y) \leq f_{y}(y)$, or $f_{y}(y) \leq \delta(\alpha, 0)$, où cette constante à été introduite en 7.1.2. $\diamond$

\subsubsection{Démonstration de la proposition 7.2 .2}

Preuve :soient $(f, S)$ et $(g, S)$ deux solutions du problème de Plateau asymptotique pour $(i, S)$. Pour tout $y \in S$, soit $D_{y}$ un disque ouvert tel que

$$
i\left(D_{y}\right) \subset \partial_{\infty} U_{\alpha_{0}}^{\left(f(y), n_{f}(y)\right)} \cap \partial_{\infty} U_{\alpha_{0}}^{\left(g(y), n_{g}(y)\right)}
$$

où $n_{f}$ et $n_{g}$ désignent les champ de vecteur normaux extérieurs associés à $f$ et $g$ respectivement. 
Soit alors $S_{y}$ l'unique solution du problème de Plateau asymptotique pour $\left(i, D_{y}\right)$ produite par le lemme 7.1.1. D'après la proposition 7.2.4, $S_{y}$ est le graphe de fonctions $f_{y}$ et $g_{y}$ au dessus de $\left(f, D_{y}\right)$ et $\left(g, D_{y}\right)$ respectivement.

Considérons un recouvrement localement fini de $S$ par des $D_{y}$ où $y$ décrit un ensemble $Y$. Et soit $f_{0}=\inf \left(f_{y}\right), y \in Y$ et $g_{0}=\inf \left(g_{y}\right], \quad y \in Y$. Par construction le graphe de $f_{0}$ et celui de $g_{0}$ sont les mêmes. Nous avons ainsi construit une surface intermédiaire $S_{2}$ qui est un graphe à la fois au dessus de $(f, S)$ et au dessus de $(g, S)$. Cette surface convexe n'a pas de raison d'être lisse.

Remplaçons cette surface $S_{2}$ par la surface $S_{3}$ à distance $r$ de de $S_{2}$, pour $r$ bien choisi, indépendamment de $S_{2}, S_{3}$ est à courbure strictement supérieure à $k$. A nouveau, $S_{3}$ est une graphe au dessus de $(f, S)$ et $(g, S)$.

Identifions comme d'habitude $S_{3}$ au disque $D_{1}$ de rayon 1 de $\mathbb{C}$. Soit $D_{r}$ les disques de rayon $r$, et $\Sigma_{r}$ les solutions du problèmes de Plateau pour $D_{r}$. Pour $r$ petit, $\Sigma_{r}$ est un graphe à la fois au dessus de $(f, S)$ et $(g, S)$. Ceci est également vrai par continuité pour tout $r$, les k-surfaces $(f, S)$ et $(g, S)$ faisant office de barrière.

Quand $r$ tend vers l'infini la suite de surfaces $\left\{\Sigma_{r}\right\}$ tend vers une k-surface $\Sigma_{0}$. Pour voir cela il suffit d'invoquer le même raisonnement qu'en 7.2.1 . .1.(iv).॰

\subsection{Existence}

Nous voulons démontrer nos théorèmes d'existence de solutions du problème de Plateau asymptotique

Théorème 7.3.1 Soit $S$ une surface compacte de genre plus grand que 2, $\rho$ une représentation de $\Gamma$ dans le groupe des isométries de $M, f$ un homéomorphisme local de $\bar{S}$, le revêtement universel de $S$ dans $\partial_{\infty} M$ équivariante sous $\rho$, alors il existe une unique solution non dégénérée du problème de Plateau asymptotique pour $(f, \bar{S})$ et cette solution est équivariante sous $\rho$.

Théorème 7.3.2 Soit $f$ un homéomorphisme local de $U$ dans $\partial_{\infty} M$ et soit $S$ un ouvert relativement compact de $U$, alors il existe une unique solution au problème de Plateau asymptotique pour $(f, S)$

Nous montrerons également ce qui peut-être conçu comme une généralisation faible du petit théorème de Picard.

Théorème 7.3.3 Soit $f$ un homéomorphisme local de $S$ dans $\partial_{\infty} M$ évitant trois points. Alors, il existe une solution non dégénérée du problème de Plateau asymptotique $\operatorname{pour}(f, S)$. 
Pour démontrer nos deux théorèmes d'existence, nous allons à chaque fois construire une surface convexe immergée dans $M$ et dont l'application de GaussMinkowski définit le un problème de Plateau asymptotique. Nous construirons cette surface en recollant des surfaces obtenues comme solution des problèmes de Plateau asymptotiques pour des disques plongés.

Les deux premiers théorèmes vont chacun utiliser des lemmes ayant leur intérêt propre. Pour le premier, il nous faudra le

Lemme 7.3.4 Soit $S$ une surface complète, qui n'est ni tubulaire, ni horosphérique à l'infini, et soit $N$ son application de Gauss Minkowski à valeurs dans $\partial_{\infty} M$, alors le problème de Plateau asymptotique $(N, S)$ admet une solution. Si de plus, $S$ n'est pas tubulaire à l'infini, cette $k$-surface n'est pas dégénérée

Pour le deuxième, nous utiliserons le

Lemme 7.3.5 Soit $V$ une surface sans bord, pas nécessairement complète, immergée de façon localement convexe dans $M$. Soit $N$ son application de GaussMinkowski à valeurs dans $\partial_{\infty}$, soit $S \subset U$ un ouvert relativement compact. alors le problème de Plateau asymptotique $(N, S)$ admet une solution.

\subsubsection{Démonstration du lemme 7.3.4}

Preuve :soit $S_{1}$ la surface à distance $r$ de $S$, cette surface n'est évidemment ni horosphérique à l'infini, ni tubulaire. Pour $r$ bien choisi, cette surface est à courbure strictement plus grande que $k$. De plus, toute solution de $\left(N, S_{1}\right)$ est une solution de $(N, S)$. Il est maintenant facile de vérifier que la $k$-surface lentille pour $S_{1}$, produite par le lemme de Morse est une solution du problème de Plateau asymptotique.»

\subsubsection{Démonstration du lemme 7.3.5}

Preuve :nous allons chercher à produire la solution du problème de Plateau asymptotique comme limite de problèmes de Plateau, comme dans la preuve de la proposition 7.2.4.

Examinons tout d'abord le cas où $S$ est un ouvert relativement compact à bord lisse. Considérons $S_{r}$ les surfaces à distance $r$ de $S$, et $\Sigma_{r}$ les surfaces lentilles solutions du problème de Plateau pour $S_{r}$. Pour adapter la démonstration de 7.2.4 il nous suffit de faire la remarque suivante. Nous savons que, par définition des surfaces lentilles $S=S_{0}$ est un graphe au dessus d'un ouvert $O$ du fibré normal étendu $N_{\Sigma_{0}}$. Si $\Sigma_{r}$ n'a pas de raisons d'être un graphe au dessus de $\Sigma_{0}$, les arguments de 7.2.1.1.(i), montre qu'elle sera un graphe au dessus de ce même ouvert $O$. 
Le reste de la preuve, c'est-à-dire les points (ii), (iii), (iv), et (v) s'adapte sans problème et nous permettent de construire une solution du problème de Plateau pour $(N, S)$.

Enfin dans le cas où $S$ n'est pas à bord lisse, il nous suffit de construire $\bar{S}$ à bord lisse relativement compact et contenant $S$. Ce que nous venons de dire nous permet de construire une solution du problème de Plateau asymptotique pour $(N, \bar{S})$ et l'application directe de la proposition 7.2.4 permet de conclure. $\diamond$

\subsubsection{Démonstration du théorème 7.3.1}

Preuve :par compacité, nous pouvons trouver des disques fermés $D_{i}, i \in J$, dont les intérieurs recouvrent $\bar{S}$ le revêtement universel de $S$, et vérifiant, si nous notons

$$
I_{i}=\left\{j \in I, D_{i} \cap D_{j} \neq \emptyset\right\}
$$

les hypothèses suivantes

(a) Pour tout $i$, le cardinal de $I_{i}$ est fini,

(b) $D_{i} \not \subset \bigcup_{j \in I_{i} \backslash\{i\}} D_{i_{j}}$,

(c) Pour tout $i$, il existe un disque $\Delta_{i}$ contenant $\bigcup_{j \in I_{i}} D_{i_{j}}$, tel que $f$ restreinte à $\Delta_{i}$ soit un plongement,

(d) la famille $\left\{D_{i}\right\}$ est $\Gamma$-équivariante.

D'après le lemme 7.1.1, nous pouvons construire des solutions $S_{j}$ de chacun des problèmes de Plateau asymptotiques défini par $\left(f, D^{j}\right)$. Chacune de ces surfaces $S_{j}$ découpe $M$ en deux parties dont l'une est convexe. Nous appellerons cette composante convexe $C_{j}$.

Remarquons maintenant que les hypothèses (a), (b) et (c) entraînent l'assertion suivante :

(e) si $j, k \in I_{i}$, alors $S_{j} \cap S_{k} \neq \emptyset$ entraîne $D_{j} \cap D_{k} \neq \emptyset$.

En effet, soit $\Sigma_{i}$ la solution du problème de Plateau asymptotique pour $\Delta_{i}$ définie par (d). D'après la proposition 7.2.4, $S_{j}$ est alors un graphe au dessus de $N^{-1}\left(D_{j}\right) \subset \Sigma_{i}$ d'où (e).

Soit maintenant

$$
U_{i}=\bigcap_{j \in I_{i}} C_{j}
$$

Cet ensemble $U_{i}$ est convexe. Son bord est la réunion de facettes. Parmi celles ci, nous distinguerons $F_{i}=S_{i} \cap \partial U_{i}$. La facette $F_{i}$ est une surface, non vide d'après 
(b) dont le bord est une réunion d'arcs $C^{\infty}$ pas nécessairement connexes, $\gamma_{(i, j)}$, $j \in I_{i}$, portions de $S_{j} \cap S_{i}$.

Plus précisément encore

$$
\gamma_{(i, j)}=S_{j} \cap S_{i} \cap U_{i}
$$

nous avons même, d'après (e)

$$
\gamma_{(i, j)}=S_{j} \cap S_{i} \bigcap_{k \in I_{i} \cap I_{j}} C_{k}
$$

En particulier $\gamma_{(i, j)}=\gamma_{(j, i)}$.

Nous pouvons maintenant recoller $F_{j}$ avec $F_{i}$ le long de $\gamma_{(i, j)}$, et obtenir ainsi de proche en proche une surface $F$ localement convexe, que nous nous empressons de lisser, de manière $C^{1}$, en prenant la surface $\Sigma$ à distance $r$, et que nous appelons $\Sigma$. Il est maintenant clair que le problème de Plateau asymptotique défini par $(N, \Sigma)$, où $N$ est l'application de Gauss-Minkowski, est équivalent à notre problème de départ.

Nous voulons appliquer notre lemme 7.3.4. Nous allons montrer que $\Sigma$ est à géométrie bornée et n'est ni tubulaire à l'infini, ni horosphérique à l'infini.

Comme l'action de $\Gamma$ sur $\Sigma$ est cocompacte, il suffit bien évidemment de montrer que $\Sigma$ n'est ni tubulaire, ni horosphérique.

Si $\Sigma$ était horosphérique, alors par définition $\Gamma$ agirait de manière cocompacte sur une horosphère. Mais ceci est impossible : avec nos hypothèses de géométrie bornée, la croissance des hororosphères est polynomiales 2.3.2 (ii).

De même, si $\Sigma$ était tubulaire, $\Gamma$ agirait de manière cocompacte sur le fibré unitaire normal à une géodésique. Ceci est impossible : ce fibré est conforme au plan privé d'un point.

Le lemme 7.3.4 permet alors de conclure.»

\subsubsection{Démonstration du théorème 7.3 .2}

Preuve :nous allons procéder comme dans le paragraphe précédent. En effet, nos hypothèses nous permettent de contruire une famille finie de disques ouverts $D_{i}$ vérifiant $i \in J$, dont les intérieurs recouvrent l'adhérence de $S$, et vérifiant, si nous notons

$$
I_{i}=\left\{j \in I, D_{i} \cap D_{j} \neq \emptyset\right\}
$$

les hypothèses suivantes

(b) $D_{i} \not \subset \bigcup_{j \in I_{i} \backslash\{i\}} D_{i_{j}}$, 
(c) Pour tout $i$, il existe un disque $\Delta_{i}$ contenant $\bigcup_{j \in I_{i}} D_{i_{j}}$, tel que $f$ restreinte à $\Delta_{i}$ soit un plongement,

D'après le lemme 7.1.1, nous pouvons construire des solutions $S_{j}$ de chacun des problèmes de Plateau asymptotiques défini par $\left(f, D^{j}\right)$. Chacune de ces surfaces

$S_{j}$ découpe $M$ en deux parties dont l'une est convexe. Nous appelerons cette composante convexe $C_{j}$.

Remarquons maintenant que les hypothèses (b) et (c) entraînent, comme dans le paragraphe précédent, l'assertion suivante :

e si $j, k \in I_{i}$, alors $S_{j} \cap S_{k} \neq \emptyset$ entraîne $D_{j} \cap D_{k} \neq \emptyset$.

En procédant exactement comme dans le paragraphe précédent, nous obtenons une surface convexe $\Sigma$ telle que le problème de Plateau asymptotique défini par $(N, \Sigma)$, où $N$ est l'application de Gauss-Minkowski, est équivalent au problème de Plateau asymptotique $\left(N, \bigcup_{i \in J} D_{i}\right)$. L'ouvert $S$ étant par hypothèse relativement compact dans $\bigcup_{i \in J} D_{i}$ ), le lemme 7.3 .5 permet de conclure.»

\subsubsection{Démonstration du théorème 7.3.3}

Preuve :soit donc $i$ un homéomorphisme local de $S$ dans $\partial_{\infty} M$ évitant trois points $x_{1}, x_{2}$ et $x_{3}$.

Pour démontrer le resultat, il nous suffit que le problème de Plateau asymptotique $\left(i, S_{0}\right)$ a une solution, où $S_{0}=\partial_{\infty} \backslash\left\{x_{1}, x_{2}, x_{3}\right\}$ et $i$ est l'injection. En effet, si cela est vrai, si $(f, S)$ est un homéomorphisme local de $S$ évitant $x_{1}, x_{2}$ et $x_{3}$, nous pouvons relever $f$ en une application $\bar{f}$ de $\bar{S}$ dans $\bar{S}_{0}$, où $\bar{S}$ et $\bar{S}_{0}$ désignent les revêtements universels de $S$ et $S_{0}$ respectivement. La proposition 7.2.4 nous permet de construire une solution du problème de Plateau asymptotique pour $(\pi \circ \bar{f}, \bar{S})$ où $\pi$ désigne la projection canonique de $\bar{S}_{0}$ dans $S_{0}$. L'unicité 7.2.1 nous permet enfin de montrer que la solution ainsi obtenue est bien une solution de $(f, S)$.

Concentrons nous donc sur le cas de $\left(i, S_{0}\right)$. Distinguons deux cas.

(i) Premièrement supposons qu'il existe un triangle idéal $T$ totalement géodésique dont les sommets sont nos trois points. La surface $S_{\epsilon}$, bord de la boule de centre $T$ et de rayon $\epsilon$ est alors à courbure plus grande que $k$ pour un certain $\epsilon$. Elle n'est de plus ni horosphérique à l'infini, ni tubulaire. Le lemme 7.3.4 nous affirme alors que la $k$-surface lentille pour $S_{\epsilon}$ produite par le lemme de Morse fournit une solution de notre problème de Plateau. Cette solution borde un convexe, elle va donc être complète, autrement dit la $k$-surface ainsi obtenue n'est pas dégénérée.

(ii) Plaçons nous dans le cas général maintenant. Soit $\left\{S_{n}\right\}_{n \in \mathbb{N}}$ une exhaustion de $S_{0}$ par des ouverts relativement compacts. Le théorème 7.3 .2 nous permet de 
construire pour tout $n$ une solution $\left(f_{n}, S_{n}\right)$ du problème de Plateau asymptotique pour $\left(i, S_{n}\right)$. Notre but est bien sûr de montrer que la suite $\left\{f_{n}\right\}_{n \in \mathbb{N}}$ converge après extraction d'une sous suite.

Soit $x$ un point appartenant à l'enveloppe convexe de $\left\{x_{1}, x_{2}, x_{3}\right\}$. Les surfaces $f_{n}\left(S_{n}\right)$ bordent des convexes $C_{n}$ qui contiennent tous $x$ et qui de plus sont tels que

$$
n \geq p \Rightarrow C_{p} \subset C_{n} \quad(*) .
$$

Considérons

$$
C=\bigcap_{n \in \mathbb{N}} C_{n}
$$

Dans le cas dans lequel nous nous sommes placé, l'intérieur de $C$ est non vide. Soit donc $x$ tel que la sphère $S_{a}$, de centre $x$ et de rayon $a$, soit incluse dans $C$. Identifions cette sphère canoniquement à $\partial_{\infty} M$.

Les surfaces $f_{n}\left(S_{n}\right)$ sont maintenant des graphes au-dessus de $S_{n}$, vus commes sous-ensembles de $S_{a}$ de fonctions $\lambda_{n}$. Par $(*)$, pour tout $y$, la suite $\left\{\lambda_{n}(y)\right\}_{n \in \mathbb{N}}$ est décroissante.

Nous pouvons en conclure, en utilisant le corollaire 4.1.3, que la suite de fonctions $\left\{\lambda_{n}\right\}_{n \in \mathbb{N}}$ converge $C_{\infty}$ vers une fonction $\lambda$ dont le graphe va nous fournir la solution de notre problème de Plateau $\left(i, S_{0}\right)$. $\diamond$

\subsection{Non existence}

Le théorème suivant montre quíl n'y a pas toujours des solutions au problème de Plateau asymptotique

Théorème 7.4.1 Si U est $\partial_{\infty} M$ privée de 0, 1 ou 2 points. Le problème de Plateau asymptotique défini par $(i, U)$, où $i$ est l'injection canonique, n'a pas de solutions.

Preuve :raisonnons par l'absurde. Dans les trois cas, la solution serait une surface $\Sigma$ globalement convexe.

Dans le premier cas, une telle surface serait une sphère compacte ce qui est clairement impossible puisque par l'équation de Gauss, la métrique induite sur une $k$-surface est à courbure négative.

Dans le deuxième cas, $\Sigma$ est une pseudo-horosphère, d'après 2.4.1 elle est alors horosphérique à l'infini. Il existe donc une suite de points $\left\{x_{n}\right\}_{n \in \mathbb{N}}$ de $\Sigma$ telle que $\left\{\left(\Sigma, x_{n}\right)\right\}_{n \in \mathbb{N}}$ converge vers une surface horosphérique $\Sigma_{0}$. Mais ceci est impossible, car par compacité $\Sigma_{0}$ est également une $k$-surface et une $k$-surface ne peut-être horosphérique à cause du principe du maximum géométrique. 
Dans le dernier cas, soit $\gamma$ la géodésique joignant les deux points du bord à l'infini et soit $\mu$ la fonction distance à cette géodésique. Soit ensuite $x_{n}$ une suite de points de $\Sigma$ tendant vers le maximum $\mu_{0}$ de $\mu$. Nous pouvons extraire une sous suite telle que $\left(\Sigma, x_{n}\right)$ converge vers $\Sigma_{0}$.

Mais $\mu_{0}$ ne peut-être borné, puisqu'alors $\Sigma_{0}$ serait tangente intérieurement à une équidistante à une géodésique, ce qui est impossible par le principe du maximum géométrique.

De même, $\mu_{0}$ ne peut-être infini, car dans ce cas la surface $\Sigma_{0}$ serait une pseudo-horosphère, et nous venons de voir que c'est impossible.»

\section{Espace des $k$-surfaces}

Abusivement, nous considérerons une $k$-surface comme une surface immergée dans le fibré unitaire de la variété $\mathrm{M}$, par son relevé de Gauss, c'est-à-dire l'application qui a un point de la surface associe sa normale extérieure.

Soyons un peu plus précis dans la définition d'une $k$-surface pour éviter le problème des revêtements multiples.

Les $k$-surfaces étant les solutions d'un problème elliptique, toute telle surface est totalement déterminée par son jet d'ordre infini en un point. On en déduit qu'étant donnée une $k$-surface $(f, S)$ sans bord, $f$ désigne une immersion de $S$ dans le fibré unitaire, il existe un représentant minimal de la $k$-surface $(g, \Sigma)$, tel que pour tout $k$-surface $(\bar{f}, \bar{S})$ ayant la même image que $f$ ( c'est-à-dire $f(S)=\bar{f}(\bar{S})$ ), alors il existe un revêtement $p$ de $\bar{S}$ sur $\Sigma$, tel que $\bar{f}=g \circ p$.

le représentant minimal d'une $k$-surface est totalement déterminé par son image, et nous ommetrons souvent de parler de l'immersion sous-jacente.

L'espace des $k$-surfaces $\mathcal{N}$ d'une variété $N$ est l'espace des paires $(S, x)$, où $x \in S$, et où $S$ est soit un tube, soit le représentant minimal $k$-surface sans bord éventuellement dégenérée.

Cet espace $\mathcal{N}$ est munie d'une topologie décrite dans [3], pour laquelle la notion de convergence est celle des sous-variétés immergées pointées décrites en 5.3.1.

Nous avons montré que dans la section 8.1 de [3] que l'espace $\mathcal{N}$ était compact et muni d'une structure de lamination, c'est à dire de produit local. Une feuille $\mathcal{L}_{S}$ de cette lamination est décrite par $S$ qui est soit le représentant minimal d'une $k$ surface, soit un tube; les points de cette feuille $\mathcal{L}_{S}$ sont alors tous les points de la forme $(S, x)$ où $x$ décrit les points de $S$. 


\section{Densité des feuilles périodiques}

A partir de maintenant, $N$ désignera une variété compacte à courbure strictement négative et $M$ sera son revêtement universel.

Soit $\mathcal{N}$ l'espace compact laminé associé décrit en 8. Nous voulons montrer

Théorème 9.0.2 L'ensemble de feuilles compactes est dense dans $\mathcal{N}$

Les feuilles compactes de $\mathcal{N}$ sont par définition les immersions localement convexes d'une surface compacte dans $N$, et dont les images sont des $k$-surfaces. Nous pouvons interpréter ces surfaces compactes dans le revêtement universel de la manière suivante. Elles s'identifient aux quadruplets $(f, \Sigma, \Gamma, \rho)$, où

(i) $\Gamma$ est un groupe discret agissant de manière cocompacte sur $\Sigma$;

(ii) $\rho$ est une représentation de $\Gamma$ dans $\pi_{1}(N)$;

(iii) $f$ est une immersion $\rho$-équivariante, localement convexe du disque $\Sigma$ dans $M$, et dont l'image est une $k$-surface.

Introduisons une autre définition, un quadruplet $(f, \Sigma, \Gamma, \rho)$ est une immersion équivariante à valeurs dans $\partial_{\infty} M$, si $\Sigma$ est une surface, éventuellement à bord, $\Gamma$ est un groupe agissant sur $\Sigma$ de facon à ce que le quotient soit une surface, $f$ est un homéomorphisme local de $\Sigma$ dans $\partial_{\infty} M, \rho$ est une représentation de $\Gamma$ dans iso $(M)$ le groupe des isométries de $M$. L'ensemble devant vérifier la relation d'équivariance c'est-à-dire

$$
\forall s \in \Sigma, \forall g \in \Gamma, f(g . s)=\rho(g) . f(s) .
$$

Une immersion équivariante est cocompacte si $\Sigma / \Gamma$ est une surface compacte.

D'après le théorème 7.3.1, les feuilles compactes de notre lamination s'identifient aux immersions équivariantes cocompactes.

Il n'est peut-être pas inutile de remarquer que dans la démonstration que nous allons donner, $\rho(\Gamma)$ sera toujours un groupe libre.

La structure de cette section est la suivante.

Dans un premier paragraphe (9.1. ), nous introduisons une définition, l'inclusion des données asymptotiques, et démontrons un lemme de compacité s'y rapportant.

Nous expliquons ensuite ( en 9.2 . ) comment associer à un plongement du disque une suite d'immersions équivariantes l'approximant en un certain sens.

Le paragraphe suivant ( 9.3 . ) montre comment construire de nouvelles immersions équivariantes à partir d'anciennes par un procédé de fusion. 
Ensuite ( en 9.4. ), nous introduisons une classe d'immersions topologiques $\mathrm{du}$ disque dans les sphères que nous appelons quasi-plongement et commençons par montrer que toute donnée asymptotique est limite de quasi-plongements.

En 9.5 . , nous montrons que tout quasi-plongement est limite de d'immersions équivariantes cocompactes : celles-ci sont obtenues par fusion d'immersions équivariantes construites en 9.2 .

Enfin, nous concluons la démonstration dans le dernier paragraphe.

\subsection{Inclusion, lemme de compacité pour les inclusions}

Dans ce qui suit, $D_{i}$ désignera toujours une variété connexe de dimension 2 sans bord.

\subsubsection{Inclusion}

Introduisons encore une définition : si $\left(f, D_{1}\right)$ et $\left(g, D_{2}\right)$ sont deux données de problèmes de Plateau asymptotiques, nous dirons que $\left(f, D_{1}\right)$ est inclus dans $\left(g, D_{2}\right)$ par $i$, et noterons

$$
\left(f, D_{1}\right) \subset\left(g, D_{2}\right) \quad[i]
$$

s'il existe un plongement $i$ de $D_{1}$ dans $D_{2}$, tel que $f=g \circ i$.

La petite observation suivante nous sera utile :

(i) soit $x_{1}$ et $x_{2}$ des points de $D_{1}$ et $D_{2}$ respectivement, si pour tout ouvert

$U$ relativement compact de $D_{1}$, nous pouvons trouver $i_{U}$ telle que $(f, U)$

est inclus dans $\left(g, D_{2}\right)$ par $i_{U}$ de telle sorte que $i_{U}\left(x_{1}\right)=x_{2}$, alors $\left(f, D_{1}\right)$

est inclus dans $\left(g, D_{2}\right)$.

\subsubsection{Intersection}

Plus généralement, si $\left\{\left(f_{n}, D_{n}\right)\right\}_{n \in \mathbb{N}}$ est une suite de problèmes de Plateau asymptotiques, si $\left(g, D_{\infty}\right)$ est un autre problème de Plateau asymptotique, inclus dans tous les $\left(f_{n}, D_{n}\right)$ par $i_{n}$, nous dirons que $\left(g, D_{\infty}\right)$ est l'intersection des $\left(f_{n}, D_{n}\right)$ le long de $i_{n}$, et nous noterons

$$
(g, D)=\bigcap_{n \in \mathbb{N}}\left(f_{n}, D\right)\left[i_{n}\right]
$$

si toute donnée $(h, U)$ ( où $U$ est connexe), incluse pour tout $n$ dans $\left(f_{n}, D_{n}\right)$ pour tout $n$ par $j_{n}$ et vérifiant de plus

$$
\left(g, D_{\infty}\right) \subset(h, U)[j]
$$


où $j$ vérifie $i_{n}=j_{n} \circ j$, alors $(h, U)=(g, D)$. On vérifie la proposition suivante

Proposition 9.1.1 Supposons que

$$
(g, D)=\bigcap_{n \in \mathbb{N}}\left(f_{n}, D\right)\left[i_{n}\right]
$$

Soit par ailleurs, $(h, U)$ telle que, pour tout $n$

$$
(h, U) \subset\left(f_{n}, D\right)\left[j_{n}\right] .
$$

Supposons qu'il existe $x_{0} \in D$ et $x_{1} \in U$ tels que pour tout $n, i_{n}\left(x_{0}\right)=j_{n}\left(x_{1}\right)$. Alors

$$
(h, U) \subset(g, D) \quad[j]
$$

de telle sorte que $j_{n}=i_{n} \circ j$.

\subsubsection{Limite inférieure}

Enfin, nous dirons que $\left(g, D_{\infty}\right)$ est la limite inférieure des $\left(f_{n}, D_{n}\right)$ par $i_{n}$ et nous noterons

$$
\left(g, D_{\infty}\right)=\liminf _{n \in \mathbb{N}}\left(f_{n}, D_{n}\right) \quad\left[i_{n}\right],
$$

si pour toutes les sous-suites $s(n)$, il existe $p$ tel que

$$
\left(g, D_{\infty}\right)=\bigcap_{n>p}\left(f_{s(n)}, D_{s(n)}\right)\left[i_{s(n)}\right]
$$

Il faut faire un petit peu attention avec ces notions : si en effet nous considérons la suite décroissante d'ouvert $\left\{U_{n}\right\}_{n \in \mathbb{N}}$ obtenue en enlevant les uns après les autres les points d'un ensemble dénombrable dont l'adhérence est un équateur, et si $f_{n}$ est la représentation conforme envoyant zéro sur le pôle nord, nous avons, suivant le choix de nos intersections deux intersections des $\left\{\left(f_{n}, U_{n}\right)\right\}_{n \in \mathbb{N}}$ : la représentation de l'hémisphère nord et celle de l'hémisphère sud.

\subsubsection{Encore un lemme de compacité}

Nous voulons montrer le 
Lemme 9.1.2 Soit $\left\{\left(f_{n}, D\right)\right\}_{n \in \mathbb{N}}$ une suite de problèmes de Plateau asymptotiques, admettant des solutions $\left\{\phi_{n}\right\}_{n \in \mathbb{N}}$ et $x_{0}$ un point de $D$; soit également une donnée asymptotique

$$
(g, D)=\liminf _{n \in \mathbb{N}}\left(f_{n}, D_{n}\right) \quad\left[i_{n}\right]
$$

Alors, la suite de $k$-surfaces $\left\{\left(\phi_{n}, D\right)\right\}_{n \in \mathbb{N}}$, pointées en $\left\{i_{n}\left(x_{0}\right)\right\}_{n \in \mathbb{N}}$, converge vers une solution du problème de Plateau asymptotique pour $(g, D)$.

Preuve :pour simplifier, nous nous noterons $0=x_{0}=i_{n}\left(x_{0}\right)$. Soit $\psi$ la solution du problème de Plateau asymptotique défini par $(g, D)$. Cette solution existe bien d'après le théorème 7.3 .2 .

Nous savons $(c f$ 7.2.4 $)$ que, pour tout $n, \psi(D)$ est un graphe au dessus d'un ouvert de $\phi_{n}(D)$.

Notre première étape consiste à démontrer l'assertion :

(1) la suite $\left\{\phi_{n}(0)\right\}_{n \in \mathbb{N}}$ reste à distance bornée de $\psi(0)$.

Raisonnons par l'absurde. Dans le cas contraire, il existe une sous-suite $s(n)$ telle que $\phi_{s(n)}(0)$ converge vers un point $m$ de $\partial_{\infty} M$.

Alors pour tout voisinage $U$ de $m$, pour $n$ suffisamment grand, si $N_{n}$ désigne l'application de Gauss-Minkowski de $\phi_{s(n)}$ et $B_{n}$ la boule de rayon 1 de centre $\phi_{s(n)}(0)$ tracée sur $\phi_{s(n)}(D)$, nous avons

$$
\partial_{\infty} M \backslash U \subset N_{n}\left(B_{n}\right) .
$$

En particulier, $\left(i, \partial_{\infty} M \backslash U\right)$ est inclus dans les $\left(f_{s(n)}, D\right)$ pour tous les $n$ suffisamment grand et ainsi dans $(g, D)$, par 9.1.1.

Maintenant, d'après notre observation préliminaire (9.1.1), nous pouvons conclure que $\left(i, \partial_{\infty} M \backslash\{m\}\right)$ est inclus dans $(g, D)$. Nous avons là notre contradiction, car le théorème 7.3 .2 fournirait alors une solution du problème de Plateau asymptotique pour $\left(i, \partial_{\infty} M \backslash\{m\}\right)$, ce qui est impossible d'après 7.4.1.

Nous avons fini de démontrer l'assertion (1).

Nous sommes maintenant en mesure d'appliquer notre théorème de compacité 4.1.1 dans notre cas particulier où il n'y a pas de condition sur le bord. Extrayons donc une sous-suite $s(n)$ telle que nous ayions l'alternative :

(a) soit $\left\{\phi_{s(n)}(D), \phi_{s(n)}(0)\right\}_{n \in \mathbb{N}}$ converge vers une $k$-surface (peut-être dégénérée) $S$,

(b) soit $\left\{n \circ \phi_{s(n)}(D), n \circ \phi_{s(n)}(0)\right\}_{n \in \mathbb{N}}$ converge vers un tube (nécessairement complet) autour d'une géodésique $\gamma$ joignant deux points à l'infini $\gamma_{+}$ et $\gamma_{-}$. Ici, bien sûr $n$ désigne, l'application de Gauss de la surface (ici, $\left.\phi_{n}(D)\right)$ à valeurs dans le fibré unitaire de $M$. 
Dans les deux cas de cette alternative, nous obtenons naturellement associée à la limite une donnée de problème de Plateau asymptotique $(f, D)$ : l'application de Gauss-Minkowski de la surface limite dans le premier cas, le revêtement universel de la sphère moins deux points dans l'autre cas.

Par convergence uniforme sur tout compact, nous en déduisons que pour tout ouvert relativement compact $U$ de $D$, nous obtenons que $(f, U)$ est inclus dans $\left(f_{s(n)}, D\right)$, pour tout $n$ suffisamment grand et donc dans $(g, D)$, par définition de limite inférieure.

Grâce à notre observation préliminaire, nous en déduisons que $(f, D)$ est inclus dans $(g, D)$.

En particulier, le deuxième cas de l'alternative est exclus : en effet, le théorème 7.3 .2 entrainerait alors une solution du problème de Plateau défini par la sphère moins deux points, ce qui est impossible par 7.4.1.

Enfin, prenons un ouvert relativement compact $U$ de $D$. Par convergence uniforme sur tout compact, nous en déduisons que $(g, U)$ est inclus dans $(f, D)$. Ainsi, $(g, D)$ est inclus dans $(f, D)$.

En conclusion, $(g, D)=(f, D)$ et, par unicité de la solution d'un problème de Plateau asymptotique 7.2.1, nous en déduisons que $\left\{\phi_{s(n)}\right\}_{n \in \mathbb{N}}$ converge vers $\psi$.

\subsection{Construction de groupes}

Nous allons dans un premier temps exhiber des groupes associés à des plongements du disque.

Pour tout $\gamma \in \pi_{1}(N)$ différent de l'identité, $\gamma^{+}$et $\gamma^{-}$désigneront respectivement les points attracteurs et répulseurs de $\gamma$ sur $\partial_{\infty} M$.

Si $F$ est un sous-groupe de $\pi_{1}(N)$, nous noterons $\Lambda(F)$ son ensemble limite et $\Omega(F)=\partial_{\infty} \backslash \Lambda(F)$ son ensemble de discontinuité. Nous dirons que $F$ est convexe cocompact si $F$ agit de manière cocompacte sur $\Omega(F)$.

La proposition que nous allons énoncer va être assez technique.

Proposition 9.2.1 Donnons nous $(f, D)$ un plongement $d u$ disque dans $\partial_{\infty} M$ et $x_{i}^{ \pm}$des points de $f(\partial D)$. Soit maintenant de plus, $\left\{\gamma_{i, n}\right\}_{n \in \mathbb{N}}, i \in\{1, \ldots, p\}, p$ suites d'éléments de $\pi_{1}(N)$, telles que

(i) les suites $\left\{\gamma_{i, n}^{ \pm}\right\}_{n \in \mathbb{N}}$ convergent vers $x_{i}^{ \pm}$,

(ii) $\forall i, n, \quad \gamma_{i, n}^{ \pm} \notin f(D)$.

Il existe alors une suite de groupes libres convexes cocompacts $\left\{F_{n}\right\}_{n \in \mathbb{N}}$ vérifiant

$$
\forall n, \quad \exists q, \quad \forall i, \quad \gamma_{i, n}^{q} \in F_{n},
$$




$$
\begin{gathered}
\forall \gamma \in F_{n} \backslash\{\operatorname{id}\}, \quad \gamma(f(D)) \cap f(D)=\emptyset \\
(f, D)=\liminf _{n \in \mathbb{N}}\left(i_{n}, \Omega\left(F_{n}\right)\right) \quad\left[f \circ i_{n}^{-1}\right],
\end{gathered}
$$

où $i_{n}$ désigne l'injection canonique de $\Omega\left(F_{n}\right)$ dans $\partial_{\infty}(M)$

Preuve :munissons $\partial_{\infty} M$ d'une métrique arbitraire, fixons un entier $n$ et soit $\varepsilon$ le réel strictement positif ( à cause de (ii)) défini par

$$
\varepsilon=\operatorname{Sup}\left\{d\left(\gamma_{i, n}^{ \pm}, x_{i}^{ \pm}\right)\right\} \text {. }
$$

Par hypothèse $\varepsilon$ tend vers 0 quand $n$ tend vers l'infini.

Par densité des géodésiques périodiques, il existe des éléments de $\pi_{1}(N)$, $\left\{\lambda_{1}, \ldots \lambda_{k}\right\}$, où $k$ est un nombre fini dépendant de $n$, tels que l'ensemble

$$
L=\left\{\lambda_{i}^{+}, \lambda_{i}^{-} ; 1 \leq i \leq k\right\}
$$

soit $\varepsilon$-proche pour la distance de Haussdorff de $f(\partial D)$ et à l'extérieur de $f(D)$. On peut enfin s'arranger pour que tous les points de $L$ soient distincts et que $l_{i}=\gamma_{i, n}$, $\forall i \in\{1, \ldots, p\}$.

Par l'argument ping-pong classique, il existe $q$ suffisamment grand tel que le groupe

$$
F_{n}=\left\langle\lambda_{1}^{q}, \ldots, \lambda_{k}^{q}\right\rangle
$$

soit libre et agisse de manière cocompacte sur son ensemble de discontinuité.

On peut de plus s'arranger en prenant $q$ suffisamment grand pour que son ensemble limite $\Lambda_{n}$ soit dans un $\varepsilon$ voisinage de $L$, et à l'extérieur de $f(D)$.

Toujours en prenant $q$ suffisamment grand, on obtient la condition (2).

Pour achever la démonstration de la proposition, il nous suffit de remarquer que $\Lambda_{n}$ est une suite de compacts convergeant pour la métrique de Haussdorff vers le bord de $f(D)$, tout en restant à l'extérieur de $f(D)$, et qu'ainsi nous avons bien

$$
(f, D)=\liminf _{n \in \mathbb{N}}\left(i_{n}, \Omega\left(F_{n}\right)\right) \quad\left[f \circ i_{n}^{-1}\right]
$$

ce que nous voulions démontrer $\diamond$

Nous avons déjà tout en main pour montrer que chaque plongement du disque est limite d'immersions équivariantes cocompactes : il nous suffit de mettre ensemble la proposition précédente et le lemme de compacité 9.1 .2

Malheureusement, toute immersion n'est pas limite de plongement. Il nous faut donc raffiner notre construction et donner une procédure, que nous appelerons fusion, permettant de construire de nouvelles immersions équivariantes cocompactes, et montrer que celle ci permet d'approximer ce que nous appelerons des quasiplongements qui eux vont s'avérer dense dans les immersions. 


\subsection{Fusion de groupes et de surfaces}

Nous allons expliquer dans ce paragraphe une construction permettant de construire de nouveaux problèmes de Plateau équivariants à partir d'anciens.

La procédure que nous allons décrire est donnée par la construction suivante. Introduisons tout d'abord nos notations et hypothèses.

Soient $\left(f_{i}, S_{i}, \Gamma_{i}, \rho_{i}\right)$, où $i \in\{1,2\}$, deux immersions équivariantes. Nous supposerons ici que les $S_{i} / \Gamma_{i}$ sont des surfaces à bord. Soient $c_{1}$ et $c_{2}$ deux composantes connexes du bord respectivement de $S_{1} / \Gamma_{1}$ et $S_{2} / \Gamma_{2}$. On suppose qu'il existe deux relevés $v_{i}$ de ces courbes dans $S_{i}$, associés à des éléments $\gamma_{i} \in \Gamma_{i}$ tels que $\rho_{1}\left(\gamma_{1}\right)=\rho_{2}\left(\gamma_{2}\right)$ ainsi qu'un homéomorphisme $j$ entre $v_{1}$ et $v_{2}$, vérifiant

$$
\forall s \in v_{2}, \quad f_{1} \circ j(s)=f_{2}(s)
$$

ainsi que

$$
\gamma_{2} \circ j=j \circ \gamma_{1}
$$

En particulier $j$ descend en un homéomorphisme $h$ entre $c_{1}$ et $c_{2}$. Notre construction découle alors de l'immédiate proposition suivante :

Proposition 9.3.1 Avec les hypothèses et notations précédentes, il existe une unique immersion équivariante $(f, \Sigma, \Gamma, \rho)$ telle que $\Sigma / \Gamma=\Sigma_{1} / \Gamma_{1} \cup_{h} \Sigma / \Gamma_{2}$ et, si $\iota_{i}$ est l'injection de $\Sigma_{i}$ dans $\Sigma$ qui se déduit de cette identification, alors $f \circ \iota_{i}=f_{i}$.

Preuve :il s'agit d'une construction standard $\diamond$

L'immersion équivariante ainsi construite sera appelée fusion des deux immersions équivariantes précédentes.

On peut remarquer alors que $\Gamma$ est un produit amalgammé de $\Gamma_{1}$ et $\Gamma_{2}$

Nous verrons plus tard comment la fusion se comporte vis-a-vis de l'opération limite inférieure.

\subsection{Quasi-plongements}

Introduisons une définition intermédiaire.

Un homéomorphisme local $f$ du disque ouvert $D$ dans $\partial_{\infty} M$ sera appelé un quasi-plongement, si $f$ se prolonge continuement en un homéomorphisme local de l'adhérence de $D$, et s'il existe une famille finie d'arcs plongés $\left\{c_{i}\right\}, 1 \leq i \leq q$ deux à deux disjoints, dont les extrémités sont dans $\partial D$, et telle que $f$ s'étend en un plongement de l'adhérence de chaque composante connexe de 


$$
D \backslash \bigcup_{1 \leq i \leq q} c_{i} .
$$

Nous appelerons la famille d'arcs de la définition, découpe du quasi-plongement.

En un certain sens, le résultat suivant affirme que tout homéomorphisme local est limite de quasi-plongements.

Proposition 9.4.1 Soit $f$ un homéomorphisme local de $D$ dans $\partial_{\infty} M$, alors pour tout ouvert relativement compact $U$ de $D$ il existe un ouvert relativement compact $V$ inclus dans $D$ contenant $U$ tel que $(f, V)$ soit un quasi-plongement

Preuve :nous allons utiliser des idées contenues dans la paramétrisation faite par Thuston de l'espace des $\mathbb{C} P^{1}$-structures par les laminations géodésiques mesurées. Notre méthode va être laborieuse, et nous aimerions savoir s'il y a plus simple.

Identifions $\partial_{\infty} M$ à la sphère $S^{2}$. Munissons $S^{2}$ d'une métrique à courbure constante et $D$ de la métrique induite par $f$. Nous noterons $\bar{D}$ sa complétion métrique et $\operatorname{Fr}(D)=\bar{D} \backslash D$. Au besoin, en restreignant un peu $D$, nous pouvons supposer que $\bar{D}$ est homéomorphe au disque fermé et que $f$ s'étend en un homéomorphisme local de $\bar{D}$ dans $S^{2}$.

Une boule métrique ouverte de $D$ sera appelée une bonne boule, si $f$ est une isométrie de cette boule sur une boule de $S^{2}$. Une boule sera maximale, si c'est une bonne boule et si elle n'est incluse dans aucune autre bonne boule qu'elle même.

Si $B$ est une boule maximale, nous noterons $\bar{B}$ son adhérence dans $D$ (et non dans $\bar{D})$ et $\operatorname{Fr}(B)$ l'ensemble $\bar{B} \backslash B$. Cet ensemble $\operatorname{Fr}(B)$ est une réunion d'intervalles ouverts. Un peu de géométrie sphérique montre que si deux points de $\operatorname{Fr}(B)$ sont dans une même composante connexe de $\operatorname{Fr}(B)$, alors ils sont inclus dans une même bonne boule.

Nous dirons enfin que deux points $x$ et $y$ de $\operatorname{Fr}(D)$ sont joignables, s'il existe une boule maximale $B$ et une composante connexe $A$ de $\operatorname{Fr}(B)$ telle que $x$ et $y$ appartiennent tous deux à l'adhérence de $A$ dans $\bar{D}$.

Reamarquons que si $x_{1}$ et $x_{2}$ sont joignables de même que $y_{1}$ et $y_{2}$ avec $y_{i} \neq x_{j}$, alors $y_{1}$ et $y_{2}$ sont tous les deux inclus dans la même composante connexe de $\operatorname{Fr}(D) \backslash\left\{x_{1}, x_{2}\right\}$. Explicitons ce raisonnement. Notons $B_{x}$ et $B_{y}$ les bonnes boules respectives pour les paires $\left(x_{1}, x_{2}\right)$ et $\left(y_{1}, y_{2}\right)$, ainsi que $A_{x}$ et $A_{y}$ les composantes connexes de $\operatorname{Fr}\left(B_{x}\right)$ et $\operatorname{Fr}\left(B_{y}\right)$ qui s'en déduisent. Il est calir que $A_{x}$ et $A_{y}$ étant des portions de cercles ne peuvent s'intersecter en une infinité de points ar lles seraient alors confondues. De plus, $y_{1}$ et $y_{2}$ sont dans des composantes connexes différentes de $\operatorname{Fr}(D) \backslash\left\{x_{1}, x_{2}\right\}$, nous en déduisons que $A_{x}$ et $A_{y}$ se rencontrent transversalement en exactement un point, puisque deux cercles ne peuvent se renconter en plus de 
deux points. Mais alors, l'une des extrémités de $A_{x}$ ( c'est-à-dire $x_{1}$ et $x_{2}$ ) serait inclus dans l'intérieur de $B_{y}$ d'où la contradiction.

Si maintenant $x$ et $y$ sont deux points joignables correspondant à une boule maximale $B$ (pas nécessairement unique d'ailleurs), traçons entre $x$ et $y$ la géodésique pour la métrique hyperbolique conforme de $B$. L'arc correspondant sera appelée bon arc. Un raisonnement géométrique élémentaire montre que deux bons arcs ne peuvent s'intersecter qu'en leurs extrémités.

La réunion $\mathcal{L}$ des bons arcs forme un ensemble fermé et nous avons construit ainsi une lamination de $D$.

Cette construction n'est bien sûr pas originale, c'est celle qui associe à toute $\mathbb{C} P^{1}$-structure une lamination comme dans la construction de Thurston déjà citée.

On remarque aisément que si $A$ est le complémentaire de $\mathcal{L}$, toute composante connexe de $A$ est inclus dans une bonne boule.

Nous pouvons maintenant construire une exhaustion de $D$ par des disques compacts $D_{n}$ telle que toute intersection non vide d'un bon arc avec $D_{n}$ soit connexe et de longueur minorée par une constante ne dépendant que de $n$ : par exemple, on peut trouver une métrique hyperbolique telle que la lamination soit géodésique, puis prendre une exhaustion par des convexes, et enfin éventuellement découper ces convexes en enlevant la "petite" composante connexe du complémentaire des bons arcs de petite longueur.

Fixons ensuite l'un des $D_{p}$; nous voulons montrer que $f$ restreinte à $D_{p}$ est un quasi-plongement.

Pour cela, il nous suffit de contruire un recouvrement de $D_{p}$ tel que tout ouvert de ce recouvrement est bordé par une courbe de Jordan contituée d'un nombre fini de bons arcs et d'arcs inclus dans le bord de $D_{p}$, et tel qu'il existe une découpe de cet ouvert par des bons arcs pour laquelle la restriction de $f$ à cet ouvert soit un quasi-plongement. En effet, lorsque l'on aura extrait un recouvrement fini par de tels ouverts, la famille de bons arcs inclus dans le bord de ces ouverts et provenant des découpes de ces mêmes ouverts, nous donnera une découpe de quasi-plongement.

Ce recouvrement est aisé à construire : nous avons quatre cas à considérer.

(1) Si $x \in A=D \backslash \mathcal{L}$, nous prenons simplement la composante connexe de $x$ dans $A \cap D_{p}$. Le longueur de la trace des bons arcs sur $D_{p}$ étant minorée, il n'y a qu'un nombre fini de bons arcs dans le bord de cette composante connexe.

(2) Si $x$ se trouve sur un bon arc isolé. Ce bon arc sépare deux composantes connexes de $A$. Nous prenons alors comme ouvert la réunion de ces composantes connexes, avec la découpe donné par le bon arc passant par $x$.

(3) Si $x$ se trouve sur un bon arc $c$ associé à une boule maximale $B$, isolé d'un côté mais pas de l'autre, c'est à dire si ce bon arc borde une composante $O$ connexe 
de $A$, et s'il existe une suite $\left\{c_{n}\right\}_{n \in \mathbb{N}}$ d'arcs distincts de $c$ tendant vers $c$. Dans ce cas, nous pouvons remarquer que pour $n$ suffisamment grand, la région $U_{n}$ bordé par $c$ et $c_{n}$ est telle que $U_{n} \cap D_{p}$ est incluse dans $B$. Nous pouvons alors prendre comme ouvert $\left(O \cup U_{n} \cup c\right) \cap D_{p}$ qui est inclus dans $B$.

(4) Le dernier cas correspond au cas $x$ appartient à un bon $\operatorname{arc} c$ et où nous pouvons trouver deux suites de bons arcs tendant vers $c,\left\{c_{n}\right\}_{n \in \mathbb{N}}$ et $\left\{g_{n}\right\}_{n \in \mathbb{N}}$, telles que la région $U_{n}$ comprise entre $c_{n}$ et $g_{n}$ contienne $c$. Il nous suffit de remarquer que pour $n$ suffisamment grand $U_{n} \cap D_{p}$ est inclus dans $B$, et de prendre enfin comme ouvert $U_{n} \cap D_{p}$.

Notre laborieuse construction est terminée. $\diamond$

\subsection{Quasi-plongements et immersions équivariantes cocom- pactes}

Le résultat essentiel de cette section est une généralisation de 9.2.1 au cas des quasi-plongements.

Proposition 9.5.1 Soit $(f, D)$ un quasi-plongement, il existe alors une suite d'immersions équivariantes cocompactes $\left\{\left(f_{n}, S_{n}\right)\right\}_{n \in \mathbb{N}}$ telles que

$$
(f, D)=\liminf _{n \in \mathbb{N}}\left(f_{n}, S_{n}\right) \quad\left[i_{n}\right]
$$

Preuve :soit donc $(f, D)$ un quasi-plongement. D'après la définition, il existe une famille finie d'arcs plongés $\left\{c_{i}\right\}, 1 \leq i \leq q$ deux à deux disjoints, dont les extrémités sont dans $\partial D$, et telle que $f$ s'étend en un plongement de l'adhérence de chaque composante connexe $D_{j}$ de

$$
D_{c}=D \backslash \bigcup_{1 \leq i \leq q} c_{i}
$$

Orientons les $\operatorname{arcs} c_{i}$, notons $x_{i}^{ \pm}$leurs extrémités et $D_{i^{ \pm}}$les composantes connexes de $D_{c}$ bordées par $c$.

Par densité des géodésiques périodiques, nous pouvons trouver des suites $\left\{\gamma_{i, n}\right\}_{n \in \mathbb{N}}$ d'éléments de $\pi_{1}(N)$ telles que

(i) les suites $\left\{\gamma_{i, n}^{ \pm}\right\}_{n \in \mathbb{N}}$ convergent vers $f\left(x_{i}^{ \pm}\right)$

(ii) $\forall i, n \quad \gamma_{i, n}^{ \pm} \notin f\left(D_{i}^{ \pm}\right)$. 
D'après le lemme 9.2.1, nous pouvons alors trouver pour toute composante $D_{j}$ de $D_{c}$ une suite de groupes libres convexes cocompacts $\left\{F_{j, n}\right\}_{n \in \mathbb{N}}$ vérifiant, si $c_{i}$ est dans le bord de $D_{j}$,

$$
\forall n, \quad \exists q, \quad \gamma_{i, n}^{q} \in F_{j, n},
$$

ainsi que

$$
\forall \gamma \in F_{j, n} \backslash\{i d\}, \quad \gamma\left(f\left(D_{j}\right)\right) \cap f\left(D_{j}\right)=\emptyset
$$

et

$$
\left(f, D_{j}\right)=\liminf _{n \in \mathbb{N}}\left(p_{j, n}, D\right)
$$

Ici $p_{j, n}$ désigne la projection du revêtement universel de $\Omega\left(F_{n}\right)$ identifié à $D$ et vérifiant $p_{n}(0)=y_{j}$, où $y_{j}$ est un point arbitraire de $D_{j}$.

Pour tout $j, n$, notons alors $\bar{S}_{j, n}$ la surface compacte

$$
\bar{S}_{j, n}=\Omega\left(F_{j, n}\right) / F_{j, n}
$$

et $\pi_{j, n}$ la projection naturelle de $\Omega\left(F_{j, n}\right)$ sur $\bar{S}_{j, n}$.

La condition (iv) nous assure que $\pi_{j, n} \circ f$ est un plongement de $D_{j}$ dans $\bar{S}_{j, n}$.

Il nous est toujours loisible dans la contruction précédente de prendre des sous-groupes d'indice finis des $F_{j, n}$. Nous avons par ailleurs une certaine liberté concernant le choix des générateurs des groupes $F_{j, n}$ autres que les $\gamma_{i}$.

Pour continuer notre démonstration, nous allons raffiner notre construction en utilisant la latitude que nous venons de décrire de telle sorte que les groupes $F_{j, n}$ vérifient les propriétés supplémentaires suivantes :

(vi) le groupe $F_{n}$ engendré par la réunion des $F_{j, n}$ est lui-même convexe cocompact,

$$
f\left(c_{i}\right) \subset \Omega\left(F_{n}\right)
$$

Choisissons maintenant, pour tout $i, n$, des voisinages $B_{i, n}^{ \pm}$de $\gamma_{i, n}^{ \pm}$, homéomorphes à la boule fermée, disjoints deux à deux, tels que

$$
\begin{gathered}
\gamma_{i, n}\left(\partial_{\infty} M \backslash B_{i, n}^{-}\right) \subset B_{i, n}^{+}, \\
\lim _{n \rightarrow \infty}\left(\operatorname{diam}\left(B_{i, n}^{ \pm}\right)\right)=0,
\end{gathered}
$$


et enfin, ces voisinages intersectent les images des $c_{i}$ exactement en leurs extrémités

$$
f\left(c_{i}\right) \cap B_{i, n}^{ \pm}=f\left(x_{i}^{ \pm}\right) .
$$

A nouveau, si besoin était, nous avons pris des sous-groupes d'indices finis $\operatorname{des} F_{j, n}$.

Nous avons presque fini nos préliminaires. Pour les terminer, nous construisons pour tout entier $n$, et tout $j \in\{1, \ldots, q\}$, une courbe $c_{i, n}$ tracée $\operatorname{sur} \Omega\left(F_{n}\right)$ telle que

$$
f\left(c_{i}\right) \subset c_{n, i}
$$

et

$$
\gamma_{i, n}\left(c_{i, n}\right)=c_{i, n}
$$

$$
c_{n, i} \backslash f\left(c_{i}\right) \subset B_{i, n}^{+} \cup B_{i, n}^{-} .
$$

Pour ce faire, il faut procéder de la manière suivante : nous savons par (viii) que $\gamma_{i, n}\left(f\left(c_{i}\right)\right) \subset B_{i, n}^{+}$. Par ailleurs, $B_{i, n}^{+} \backslash B_{i, n}^{-}$est connexe de même que son adhérence $A$. Par $(\mathrm{x})$, cette adhérence $A$ intersecte $f\left(c_{i}\right)$ exactement en $f\left(x_{i}^{+}\right)$et $\gamma_{i, n}\left(f\left(c_{i}\right)\right)$ en $\gamma_{i, n}\left(f\left(x_{i}^{-}\right)\right)$. Choisissons donc un chemin plongé $\lambda_{i, n}$ joignant $f\left(x_{i}^{+}\right)$et $\gamma_{i, n}\left(f\left(x_{i}^{-}\right)\right)$ dans $A$. Nous pouvons alors prendre comme courbe $c_{i, n}$, la réunion des images itérées de l'arc $f\left(c_{i}\right) \cup \lambda_{i, n}$ :

$$
c_{i, n}=\bigcup_{p \in \mathbb{Z}} \gamma_{i, n}^{p}\left(f\left(c_{i}\right) \cup \lambda_{i, n}\right) .
$$

(xiv) Enfin, en prenant éventuellement des sous-groupes d'indice fini des $F_{j, n}$, la condition (xiii) et le fait que les $B_{i, n}^{ \pm}$sont deux à deux disjoints, nous pemettent d'assurer que, pour tout $j$ et $n$, si $c_{i_{1}}, \ldots c_{i_{m}}$, sont tracées dans le bord de $D_{j}$, alors les courbes $\pi_{j, n}\left(c_{i_{k}, n}\right)$ sont des courbes plongées et d'intersection vide dans $\bar{S}_{j, n}=\Omega\left(F_{n}\right) / F_{n}$.

Rappelons qu'ici $D_{j}$ est une composante connexe de

$$
D \backslash \bigcup_{1 \leq i \leq q} c_{i} .
$$

Nous avons maintenant fini d'imposer des conditions supplémentaires aux groupes $F_{j, n}$ et pouvons continuer notre démonstration. Par la condition (iv), $\pi_{j, n} \circ f$ 
est un plongement de $D_{j}$ dans

$$
U_{j, n}=\bar{S}_{j, n} \backslash \bigcup_{1 \leq k \leq m} \pi_{j, n}\left(c_{i_{k}, n}\right) .
$$

Notons donc $\Sigma_{j, n}$ la composante connexe de $U_{j, n}$ dans laquelle s'envoie $D_{j}$. Par construction, $\Sigma_{j, n}$ est une surface dont le bord s'identifie à la réunion des courbes $\pi_{j, n}\left(c_{i_{k}, n}\right)$. Soit enfin $\Gamma_{j, n}$ le groupe fondamental de $\Sigma_{j, n}$, et $S_{j, n}$ son revêtement universel.

Notre étape suivante va être de fusionner toutes les surfaces $S_{j, n}$. La procédure est la suivante : pour tout $i$ et $n$, la construction décrite en 9.3, nous permet précisemment de fusionner $S_{n}^{i^{+}}$et $S_{n}^{i^{-}}$le long de $c_{i, n}$. En procédant de proche en proche, nous produisons ainsi une immersion équivariante cocompacte $\left(f_{n}, S_{n}, \Gamma_{n}\right)$ ayant la propriété suivante : il existe un plongement $i_{n}$ de $D$ dans $S_{n}$ tel que $f=f_{n} \circ i_{n}$ c'est-à-dire

$$
(f, D) \subset\left(f_{n}, S_{n}\right)\left[i_{n}\right] .
$$

Par ailleurs les courbes $c_{i, n}$ donnent naissance à des courbes $q_{i, n}$ tracées sur $S_{n}$ et telles que $i_{n}\left(c_{i}\right) \subset q_{i, n}$ ainsi que $f_{n}\left(q_{i, n}\right)=c_{i, n}$.

Pour conclure, nous voulons montrer

$$
(f, D)=\liminf _{n \in \mathbb{N}}\left(f_{n}, S_{n}\right) \quad\left[i_{n}\right] .
$$

Soit donc $(g, U)$ un plongement d'un ouvert $U$ connexe, tel que

$$
(g, U) \subset\left(f_{s(n)}, S_{s(n)}\right)\left[j_{s(n)}\right],
$$

et

$$
(f, D) \subset(h, U) \quad[j],
$$

avec $i_{n}=j_{n} \circ j$.

Pour simplifier les notations déjà lourdes, nous allons supposer $s(n)=n$ ce qui est indolore.

Nous supposerons, en considérant $j$ comme une inclusion, que $D$ est un ouvert de $U$ et notons $Z(D)$ la frontière de $D$ dans $U$. Nous voulons montrer que $Z(D)$ est vide. Raisonnons par l'absurde et supposons que cet ensemble est non vide.

Munissons $\partial_{\infty} M$ d'une métrique annexe, $U, S_{n}$ et $D$ des métriques induites. Par notre hypothèse (ix),

$$
\lim _{n \rightarrow \infty}\left(\operatorname{diam}\left(B_{i, n}^{ \pm}\right)\right)=0,
$$


ce qui nous permet de trouver un point $x$ de $Z(D)$ et un nombre $\varepsilon>0$ tel que

$$
\forall i, n \quad d\left(g(x), B_{i, n}^{ \pm}\right) \geq \varepsilon .
$$

Nous pouvons également nous assurer que

$$
\forall i, d\left(x, c_{i}\right)_{g} e q \varepsilon
$$

et en particulier, à cause de (xiii)

$$
\text { (xv). }
$$

$$
\forall i, n \quad d\left(i_{n}(x), q i, n\right) \geq \varepsilon .
$$

En prenant $\varepsilon$ suffisamment petit, nous pouvons de plus assurer que $g$ est une bijection de la boule $B_{\varepsilon}^{U}$ de rayon $\varepsilon$ de centre $x$ dans $U$ et la boule $B_{\varepsilon}$ de centre $g(x)$ de rayon $\varepsilon$ dans $\partial_{\infty} M$. de

Le point $x$ appartient nécessairement à la frontière d'une des composantes $D_{j}$

$$
D \backslash \bigcup_{1 \leq i \leq q} c_{i} .
$$

Rappelons maintenant que $S_{n}$ est la réunion de copies isométriques des surfaces $S_{j, n}$, bordées par les courbes $q_{i, n}$ qui s'envoient par $f_{n}$ sur les courbes $c_{i, n}$.

La condition (xv) nous permet d'affirmer que $j_{n}\left(B_{\varepsilon}^{U}\right)$ est inclus dans l'intérieur d'une de ces copies de $S_{j, n}$. En particulier, puisque

$$
B_{\varepsilon}=g\left(B_{e}^{U}\right)=f_{n} \circ j_{n}\left(B_{e}^{U}\right),
$$

nous en déduisons que, pour tout $n, g\left(B_{\varepsilon}\right)$ est inclus dans $\Omega\left(F_{j, n}\right)$, ce qui est impossible car la limite de Haussdorff des $\Lambda\left(F_{j, n}\right)$ contient $\operatorname{Fr}\left(D_{j}\right)$, et en particulier $g(x)$, par construction.

Ce dernier point est la contradiction recherchée.»

\subsection{Démonstration du théorème 9.0 .2}

Nous pouvons maintenant démontrer la densité des feuilles périodiques dans l'espace laminé $\mathcal{M}$.

Tout d'abord la proposition 9.5.1 et le lemme 9.1.2 entraîne que l'adhérence de l'ensemble des feuilles périodiques contient l'ensemble des quasi-plongements.

Le théorème 9.0 .2 suit alors de la proposition 9.4.1 et de la proposition suivante 
Proposition 9.6.1 Soit $(f, D)$ une donnée asymptotique, soit $\{U\}_{n \in \mathbb{N}}$ une suite emboitée d'ouvert relativement compacts de $D$ tel que

$$
\bigcup_{n \in \mathbb{N}} U_{n}=D
$$

Notons $\left\{\left(\phi_{n}, U_{n}\right)\right\}_{n \in \mathbb{N}}$ la suite de solutions des problèmes de Plateau asymptotiques définis par $\left\{\left(f, U_{n}\right)\right\}_{n \in \mathbb{N}}$ et obtenues par le théorème 7.3.9. Nous avons alors les deux résultats suivants :

(i) si $(f, D)$ admet une solution $(\phi, D)$ alors la suite $\left\{\left(\phi_{n}, U_{n}\right)\right\}_{n \in \mathbb{N}}$ pointées en l'origine de $D$ converge vers $(\phi, D)$.

(ii) si $D$ est le revêtement universel $\partial_{\infty} M$ auquel on a ôté les deux extrémités d'une géodésique $\gamma$, et si $f$ est la projection canonique, alors la suite de surfaces $\left\{S_{n}\right\}_{n \in \mathbb{N}}$, immergées dans $U M-$ où $S_{n}$ est l'ensemble des vecteurs normaux extérieurs à la surface $\phi_{n}\left(U_{n}\right)$ - pointées en l'origine de $D$, converge vers le tube de $\gamma$.

Preuve :démontrons (i) tout d'abord. Nous allons ressasser nos idées habituelles. D'après 7.2.1, pour tout $n, \phi_{n}\left(U_{n}\right)$ est un graphe d'une fonction $f_{n}$ au dessus de $\phi\left(U_{n}\right)$ . D'aprés le lemme 7.1 .2

$$
\forall x \in D, \exists K, \text { s.t. } \forall n, \quad f_{n}(x) \leq K .
$$

Nos arguments de compacité montre que la suite de fonctions $f_{n}$ converge alors $C^{\infty}$ sur tout compact. Le graphe de la limite est alors nécessairement une solution du problème de Plateau asymptotique défini par $(f, D)$. L'unicité de la solution du problème de Plateau asymptotique, montre que cette fonction limite est nulle et donc (i).

En ce qui concerne (ii), il nous suffit de montrer que quelle que soit la soussuite $s(n),\left\{\left(\phi_{s(n)}, U_{s(n)}\right)\right\}_{n \in \mathbb{N}}$ pointées en l'origine de $D$ ne converge pas vers une $k$ surface. En effet dans ce cas, la suite $\left\{S_{n}\right\}_{n \in \mathbb{N}}$ converge vers le tube d'une géodésique d'après 4.1.1, et par construction de $D$, cette géodésique ne peut être que $\gamma$. Or si $\left\{\left(\phi_{s(n)}, U_{s(n)}\right)\right\}_{n \in \mathbb{N}}$ pointées en l'origine de $D$ ne convergeait vers une $k$-surface, celle ci seriat une solution du probleme de Plateau asymptotique pour $\partial_{\infty} M$ auquel on ôté un ou deux points et d'après 7.4.1 une telle solution n'existe pas.»

\subsubsection{Remarque}

Si $(f, D)$ est une donnée asymptotique qui s'étend en un homeomorphisme d'un voisinage du disque fermé dans $\partial_{\infty} M$, et $(\phi, D)$ est une $k$-surface solution du 
problème de Plateau asymptotique pour $(f, D)$, alors $(\phi, D)$ n'est pas tubulaire à l'infini. En effet, si $(\phi, D)$ était tubulaire à l'infini, alors il existerait une suite $\left\{x_{n}\right\}_{n \in \mathbb{N}}$ de points de $\partial_{\infty} M$, telle que le cardinal de $f^{-1}\left(x_{n}\right)$ tende vers l'infini.

\subsection{Feuilles périodiques de même genre}

Nous voulons démontrer le

Proposition 9.7.1 Soit $\left\{\Theta_{n}\right\}_{n \in \mathbb{N}}=\left\{\left(f_{n}, S_{n}, \Gamma_{n}, \rho_{n}\right)\right\}_{n \in \mathbb{N}}$ une suite d'immersions équivariantes cocompactes telle que le genre de $S_{n} / \Gamma_{n}$ soit borné. Soit $\left\{x_{n}\right\}_{n \in \mathbb{N}}$ une suite de points telle que $x_{n} \in S_{n}$ et que $\left\{f_{n}\left(x_{n}\right)\right\}_{n \in \mathbb{N}}$ reste dans un compact.

Supposons que $\left\{\left(f_{n}, S_{n}, x_{n}\right)\right\}_{n \in \mathbb{N}}$ converge vers $\left(f_{\infty}, S_{\infty}, x_{\infty}\right)$, nous avons alors les possibilités suivantes :

(i) soit il existe $\Gamma_{\infty}$ et $\rho_{\infty}$ telle que $\left(f_{\infty}, S_{\infty}, \Gamma_{\infty}, \rho_{\infty}\right)$ est cocompacte et auquel cas $\left\{\Theta_{n}\right\}_{n \in \mathbb{N}}$ est constante à partir d'un certain rang,

(ii) soit $\left(f_{\infty}, S_{\infty}\right)$ est dégénérée ou tubulaire à l'infini.

Remarquons qu'il découle de 9.6.1 et 9.6.1 que l'ensemble des $k$-surfaces qui ne sont ni dégénérées, ni tubulaires à l'infini est dense dans l'espace $\mathcal{N}$. Par ailleurs, a cause de l'unicite dans le lemme de Morse, l'ensemble des $k$-surfaces compactes de même genre est discret. Nous en déduisons un raffinement de 9.0 .2 :

Théorème 9.7.2 Soit $g \in \mathbb{N}$, alors l'ensemble des feuilles compactes de genre plus grand que $g$ est dense dans $\mathcal{N}$

\subsubsection{Démonstration de 9.7.1}

Montrons tout d'abord la

Proposition 9.7.3 Soit $S$ une $k$-surface à courbure moyenne bornée, alors il existe une constante $\varepsilon$ strictement positive telle que, pour tout $x \in S$, le rayon d'injectivité de $S$ en $x$ pour la métrique induite de celle de $N$ est minoré par $\varepsilon$

Preuve :la démonstration est immédiate. La courbure de $S$ étant uniformément bornée, le théorème de compacité 4.1.1 nous assure que $S$ est à géométrie bornée. Le rayon d'injectivité est donc bien uniformément minoré.»

Nous pouvons maintenant démontrer le théorème 9.7.2. D'après l'équation de Gauss, la courbure des métriques sur $S_{n}$ est coincée entre deux constantes strictement négatives. 
Si le diamètre de $S_{n} / \Gamma_{n}$ est unformément borné, nous en déduisons que $S_{n} / \Gamma_{n}$ converge vers une surface compacte, et que nécessairement la limite de

$$
\left\{\left(f_{n}, S_{n}, \Gamma_{n}, \rho_{n}\right)\right\}_{n \in \mathbb{N}}
$$

est une immersion équivariante cocompacte. En particulier, nous en déduisons que $\left\{\left(\Gamma_{n}, \rho_{n}\right)\right\}_{n \in \mathbb{N}}$ devient constante à partir d'un certain rang, et ceci entraîne $\left\{\Theta_{n}\right\}_{n \in \mathbb{N}}$ elle même est constante à partir d'une certain rang, par le lemme de Morse.

Si le diamètre de $S_{n} / \Gamma_{n}$ tend vers l'infini, nous en déduisons que le rayon d'injectivité de $S_{\infty}$ est nul. Par 9.7.3, la courbure moyenne de $f_{\infty}$ n'est pas bornée. $\left(f_{\infty}, S_{\infty}\right)$ est donc dégénérée ou tubulaire à l'infini.»

\section{Généricité des feuilles denses}

Nous nous proposons de démontrer

Théorème 10.0.4 L'ensemble des points de $\mathcal{N}$ par lesquel passent des feuilles denses est une intersection dénombrable d'ouverts denses

Preuve :nous allons en fait démontrer une résultat plus fort. Notons $\mathcal{M}$ l'espace laminé associé à $M$, c'est-à-dire tel que $\mathcal{N}=\mathcal{M} / \pi_{1}(N)$, alosr, déjà dans $\mathcal{M}$ une feuille générique est dense.

Remarquons tout d'abord que l'espace $\mathcal{N}$ posséde une base dénombrable d'ouverts. Pour cela, il suffit de trouver une application continue injective de $\mathcal{N}$ dans un espace à base dénombrable. Notons donc $N(k)$, l'espace des $k$-jets de plans de $N$, et $N(\infty)$ la limite projective des $N(k)$. Cet espace est à base dénombrable et l'application naturelle de $\mathcal{N}$ dans $N(\infty)$ est injective, par ellipticité. Nous en déduisons bien sûr que $\mathcal{M}$ est lui aussi à base dénombrable.

Pour conclure, il nous faut donc montrer que si $x$ et $y$ sont deux points de $\mathcal{M}$, il existe deux suites de points $\left\{x_{n}\right\}_{n \in \mathbb{N}}$ et $\left\{y_{n}\right\}_{n \in \mathbb{N}}$, tendant respectivement vers $x$ et $y$, telles que pour tout $n, x_{n}$ et $y_{n}$ soient sur la même feuille $\mathcal{L}_{n}$.

Nous nous donnons donc deux $k$-surfaces pointées $\left(f_{\infty}, S, x\right)$ et $\left(\phi_{\infty}, \Sigma, y\right)$, dans $M$; notons $f$, et $\phi$ respectivement, leurs applications de Gauss-Minkowski à valeurs dans $\partial_{\infty} M$.

Soit maintenant $\left\{S_{n}\right\}_{n \in \mathbb{N}}$, et $\{\Sigma\}_{n \in \mathbb{N}}$ des exhaustions de $S$ et $\Sigma$ par des ouverts relativement compacts, contenant $x$ et $y$ respectivement.

Soit alors $\left(f_{n}, \Sigma_{n}\right)$, et $\left(\phi_{n}, \Sigma_{n}\right)$, les solutions des problèmes de Plateau asymptotiques définis respectivement par $\left(f, \Sigma_{n}\right)$, et $\left(\phi, \Sigma_{n}\right)$. 
D'après la proposition 9.6.1, $\left\{\left(f_{n}, \Sigma_{n}, x\right)\right\}_{n \in \mathbb{N}}$, et $\left\{\left(\phi_{n}, \Sigma_{n}, y\right)\right\}_{n \in \mathbb{N}}$, convergent respectivement vers $\left(f_{\infty}, \Sigma, x\right)$ et $\left(\phi_{\infty}, \Sigma, y\right)$.

Par ailleurs, pour tout entier $p$, nous pouvons construire une famille de données asymptotiques $\left\{\left(h_{(p, n)}, U_{(p, n)}\right\}_{n \in \mathbb{N}}\right.$ et des injections $i_{(p, n)}$ et $j_{(p, n)}$ de $S_{p}$ et $\Sigma_{p}$ dans $U_{(p, n)}$ respectivement, telles que l'on ait

$$
\left(f_{p}, S_{p}\right)=\liminf _{n \in \mathbb{N}}\left(h_{(p, n)}, U_{(p, n)}\right) \quad\left[i_{(p, n)}\right],
$$

ainsi que

$$
\left(\phi_{p}, S_{p}\right)=\liminf _{n \in \mathbb{N}}\left(h_{(p, n)}, U_{(p, n)}\right) \quad\left[j_{(p, n)}\right] .
$$

Donnons l'esquisse de cette construction, il suffit de rejoindre $f\left(S_{p}\right.$ et $\phi\left(S_{p}\right)$ respectivement par des rubans dont l'épaisseur tend vers 0 quand $n$ tend vers l'infini.

Nous pouvons enfin nous débrouiller pour que, pour tout $n$ et $p$, le problème de Plateau asymptotique $\left(h_{(p, n)}, U_{(p, n)}\right)$ possède une solution $\left(H_{(p, n)}, U_{(p, n)}\right)$.

D'après le lemme 9.1.2, la suite de $k$-surfaces pointées

$$
\left\{\left(H_{(p, n)}, U_{(p, n)}, i_{(p, n)}(x)\right)\right\}_{n \in \mathbb{N}}
$$

converge vers $\left(f_{p}, S_{p}, x\right)$, et, respectivement, la suite

$$
\left\{\left(H_{(p, n)}, U_{(p, n)}, j_{(p, n)}(y)\right)\right\}_{n \in \mathbb{N}}
$$

converge vers $\left(\phi_{p}, S_{p}, y\right)$

Nous pouvons donc trouver une sous-suite $s(n)$ telle que les suites de $k$ surfaces pointées

$$
\left\{\left(H_{(s(n), n)}, U_{(s(n), n)}, i_{(s(n), n)}(x)\right)\right\}_{n \in \mathbb{N}}
$$

et

$$
\left\{\left(H_{(s(n), n)}, U_{(s(n), n)}, j_{(s(n), n)}(y)\right)\right\}_{n \in \mathbb{N}}
$$

convergent respectivement vers $\left(f_{\infty}, S, x\right)$ et $\left(\phi_{\infty}, \Sigma, y\right)$.

En enonçant ceci dans le cadre de $\mathcal{N}$, les suites de points de $\mathcal{N}$ définies par

$$
\left\{X_{n}\right\}_{n \in \mathbb{N}}=\left\{\left(H_{(s(n), n)}, U_{(s(n), n)}, i_{(s(n), n)}(x)\right)\right\}_{n \in \mathbb{N}}
$$

et

$$
\left\{Y_{n}\right\}_{n \in \mathbb{N}}=\left\{\left(H_{(s(n), n)}, U_{(s(n), n)}, j_{(s(n), n)}(y)\right)\right\}_{n \in \mathbb{N}},
$$

convergent vers $X=\left(f_{\infty}, S, x\right)$ et $Y=\left(\phi_{\infty}, \Sigma, y\right)$. Par ailleurs, pour tout $n, X_{n}$ et $Y_{n}$ appartiennent à la même feuille $\mathcal{L}_{n}$, définie par

$$
\mathcal{L}_{n}=\left(H_{(s(n), n)}, U_{(s(n), n)}\right) .
$$

C'est ce que nous voulions démontrer.» 


\section{Stabilité}

Notre but dans cette section est de démontrer un analogue du théorème de stabilité pour le flot géodésique des variétés à courbure strictement négative :

Théorème 11.0.5 Soit $N$ une variété compacte de dimension 3, sig est une métrique à courbure strictement plus petite que -1 , et $k \in] 0,1\left[\right.$, nous noterons $\mathcal{N}_{g}^{k}$, son espace des $k$-surfaces convexes.

Si $k$ et $l$ appartiennent à ]0,1[, si g et h sont deux métriques appartenant à la même composante connexe de l'espace des métriques à courbure plus petite que-1, il existe alors un homéomorphisme $\Phi$ de $\mathcal{N}_{g}^{k}$ dans $\mathcal{N}_{h}^{l}$ envoyant feuille sur feuille.

\section{$11.1 \quad$ Un lemme}

Nous voulons montrer le

Lemme 11.1.1 Soitg une métrique à courbure strictement négative sur $N$ et c une constante strictement positive, il existe un voisinage $U$ de g pour la topologie $C^{\infty}$ tel que, $\forall h, \bar{h} \in U$, pour toute surface immergée complète à géométrie bornée $(f, S)$ dont les courbures principales (pour la métrique $g$ ) sont plus grandes que $c$, si $(f, S)$ est horosphérique à l'infini pour $h$, alors $(f, S)$ est horosphérique à l'infini pour $\bar{h}$.

Preuve :on peut choisir $U$ pour que $(f, S)$ ait une courbure plus grande que $c^{2} / 4$ pour toutes les métriques de $U$, et, en particulier, soit une surface convexe.

Pour montrer le lemme, remarquons qu'il nous suffit de montrer que si $(f, S)$ est horosphérique pour $h$, alors $(f, S)$ est horosphérique à l'infini pour $\bar{h}$. Or si, $(f, S)$ est horosphérique, elle (ou plus exactement son image inverse dans le revêtement universel) borde un ensemble convexe ayant exactement un point à l'infini. Le bord à l'infini étant un invariant de quasi-isométrie, nous en déduisons que le convexe bordé par $(f, S)$ à lui aussi un seul point à l'infini pour $\bar{h}$.

La surface $(f, S)$ est donc une pseudo-horosphère pour la métrique $g$ et par le lemme 2.4.1, elle est horosphérique à l'infini pour $\bar{h} . \diamond$

\subsubsection{Démonstration du théorème 11.0.5}

Il nous suffit bien sûr de montrer le théorème pour deux métriques $\bar{h}$ et $h$ suffisamment proches au sens $C^{\infty}$. Nous allons les prendre dans un ouvert $U$ donné par lemme précédent pour une constante $c$ adéquate. 
Soit tout $\mathcal{L}=(f, S)$ une feuille de $\mathcal{N}_{\bar{h}}^{k}$. Nous voyons ici $(f, S)$ comme une immersion de $S$ dans $U N$ le fibré unitaire de $N$. Notons alors $f_{R}$ l'application de $S$ dans $N$, donnée par

$$
f_{R}: s \mapsto \exp (R f(s)) .
$$

Nous savons ( $c f$ corollaire 3.1.2), que, pour $R$ choisi suffisamment grand indépendamment de $S$, chacune des courbures principales de $f_{R}$ est plus grande qu'une constante $c$, avec $c>l^{1 / 2}$,

Nous en déduisons que si $h$ est une métrique suffisamment proche de $\bar{h}$, l'immersion $\left(f_{R}, S\right)$ est pour cette métrique $h$ à courbure plus grande que $l$.

Nous sommes en mesure de construire notre homéomorphisme $\Phi$. Séparons en deux cas,

(i) Si $(f, S)$ est un tube alors, par le lemme de Morse pour les géodésiques, $\left(f_{R}, S\right)$ est tubulaire pour la métrique $h$, soit $\gamma$ la géodésique correspondante et $T$ son tube. Nous avons donc une projection radiale naturelle $\pi$ de $f_{R}(S)$ sur $T$. Nous posons alors si $x \in S$

$$
\Phi(f, S, x)=\left(T, \pi \circ f_{R}(x)\right),
$$

où nous voyons maintenant $(f, S, x)$, resp. $\left(T, \pi \circ f_{R}(x)\right)$, comme un point de $\mathcal{N}_{\bar{h}}^{k}$, resp. de $\mathcal{N}_{h}^{l}$.

(ii) $\mathrm{Si}(f, S)$ n'est pas un tube, $\left(f_{R}, S\right)$ n'est pas tubulaire pour $h$ ( par le lemme de Morse pour les géodésiques ). De plus, par le lemme 11.1.1 $\left(f_{R}, S\right)$ n'est pas horosphérique à l'infini. Notre lemme de Morse pour les surfaces convexes 6.0.2, nous permet alors de construire une $l$-surface lentille pour la métrique $h,(\bar{f}, \bar{S})$ pour $\left(f_{R}, S\right)$. Enfin, notons $\pi$ la projection qui a tout point de $\left(f_{R}, S\right)$ associe son pied $\operatorname{sur}(\bar{f}, \bar{S})$. Nous pouvons alors définir

$$
\Phi(f, S, x)=\left(\bar{f}, \bar{S}, \pi \circ f_{R}(x)\right) .
$$

A nouveau, nous voyons $(f, S, x)$, resp.$\left(\bar{f}, \bar{S}, \pi \circ f_{R}(x)\right)$, comme un point de $\mathcal{N}_{\bar{h}}^{k}$, resp. de $\mathcal{N}_{h}^{l}$.

Par construction, $\Phi$ envoie feuille sur feuille. La continuité de $\Phi$ provient de 4.0 .1 .

Pour démontrer, que $\Phi$ est une bijection, remarquons tout d'abord que $\Phi$ envoie bijectivement chaque feuille sur chaque feuille. Soit maintenant $\bar{\Phi}$ l'application obtenue en inversant les rôles de $h$ et $\bar{h}$. L'unicité dans le lemme de Morse permet de montrer $\Phi \circ \bar{\Phi}$ envoie chaque feuille dans elle même. L'application $\Phi \circ \bar{\Phi}$ est donc une bijection, ce qui entraîne que $\Phi$ elle-même est une bijection. $\diamond$ 


\section{Références}

[1] M. Anderson The Dirichlet Problem at infinity for manifolds of negative curvatureJ. Differ. Geom. 18, 701-722 (1983)

[2] M. Gromov Foliated Plateau Problem I G.A.F.A. 1, 14-79 (1991)

[3] F. Labourie Equations de Monge-Ampère, courbes holomorphes et laminations G.A.F.A. 7, 496-534 (1997)

[4] F. Labourie Surfaces convexes dans l'espace hyperbolique et $\mathbb{C P}^{1}$ structures Jour. Lond. Mat. Soc. 111, 549-565 (1992)

[5] H. Rosenberg, J. Spruck On the existence of convex hypersurfaces of constant Gauss curvature in hyperbolic space J. Differ. Geom. 40-2, 379409 (1994). 